\title{
A Theoretical Study of the Photophysics of 8-Vinylguanine, an Isomorphic Fluorescent Analogue of Guanine
}

\author{
Electronic Supporting Information
}

\author{
Michał A. Kochman,,$^{* \dagger}$ Martina Pola, ${ }^{\dagger, \ddagger}$ R. J. Dwayne Miller,,
}

$\dagger$ Max Planck Institute for the Structure and Dynamics of Matter and Hamburg Centre for Ultrafast Imaging, Bldg. 99 (CFEL), Luruper Chaussee 149, 22761 Hamburg, Germany.

‡ Institut für Theoretische Physik, Universität Hamburg, Jungiusstraße 9, 20355 Hamburg, Germany.

ฯ Department of Chemistry and Physics, University of Toronto, 80 St. George Street, Toronto, Ontario M5S 3H6, Canada.

E-mail: michal.kochman@mpsd.mpg.de

\section{Verification of the accuracy of the MP2/ADC(2) method}

The present section of the Supporting Information provides an analysis of the realism of the $\mathrm{MP} 2 / \mathrm{ADC}(2)$ combination of methods for various aspects of the photochemistry of 9H-guanine and 8-vinylguanine $(8 \mathrm{vG})$.

\subsection{Excited electronic states of 8vG}

Our study revolves primarily around the structure and properties of the excited electronic states of $8 \mathrm{vG}$. The realism of our electronic structure method of choice - the computationally efficient MP2/ADC(2) combination - for this molecule was assessed through comparison with reference provided by the method of equations-of-motion coupled cluster with single and double excitations (EOM-CCSD). ${ }^{1}$ Such an assessment is necessitated by the potentially unbalanced treatment of $\mathrm{n} \pi^{*}$ - and $\pi \pi^{*}$-type excited states by the $\operatorname{ADC}(2)$ method. The benchmark studies by Szalay and coworkers $^{2}$ and Kánnár and Szalay ${ }^{3}$ have demonstrated that the second-order approximate coupled cluster (CC2) method (to which $\mathrm{ADC}(2)$ is closely related ${ }^{4}$ and similar in terms of accuracy) often underestimates vertical excitation energies into $n \pi^{*}$-type states while, in contrast, vertical excitation energies into $\pi \pi^{*}$-type states tend to coincide closely with the reference provided by the method of equation of motion coupled-cluster methods including perturbative triple excitations $(\operatorname{EOM}-\operatorname{CCSD}(\mathrm{T}))$. The net outcome is a somewhat unbalanced description of $\mathrm{n} \pi^{*}$ - and $\pi \pi^{*}$-type states by the $\mathrm{CC} 2$ method; this conclusion may be extended to the MP2/ADC(2) combination. In turn, the EOM-CCSD method systematically overestimates vertical excitation energies into states of all types by 0.1 to $0.3 \mathrm{eV}$, but without bias towards excited states of a specific character. ${ }^{2,3}$ Therefore, although EOM-CCSD cannot be expected to yield accurate vertical excitation energies, it should provide a reliable benchmark for the relative energy ordering of the low-lying excited states of $8 \mathrm{vG}$.

In order to enable comparison with the MP2/ADC $(2)$ method, the vertical excitation spectrum of $8 \mathrm{vG}$ was calculated at the EOM-CCSD/cc-pVDZ level of theory, as well as at the MP2/ADC(2)/cc-pVDZ 
Table S1: Vertical excitation energies $(\Delta E)$, oscillator strengths $(f)$ and electric dipole moments $(\mu)$ of $8 \mathrm{vG}$ at its ground-state equilibrium geometry, calculated at the MP2/ADC(2)/cc-pVDZ and EOM-CCSD/cc-pVDZ levels of theory. $\Delta q_{\mathrm{v}}$ denotes the amount of charge donated onto the vinyl group in the ICT state.

\begin{tabular}{|l|l|l|l|l|l|}
\hline Level of theory & State & $\Delta E, \mathrm{eV}$ & $f$ & $\mu, \mathrm{D}$ & $\Delta q_{\mathrm{v}}, e$ \\
\hline MP2/ADC(2)/cc-pVDZ & $\mathrm{S}_{0}$ & & & 5.69 & \\
& $\mathrm{~S}_{1}\left(\mathrm{ICT} \pi \pi^{*}\right)$ & 4.696 & 0.683 & 8.27 & -0.185 \\
& $\mathrm{~S}_{2}\left(\mathrm{~L}_{\mathrm{a}} \pi \pi^{*}\right)$ & 5.004 & 0.062 & 5.82 & \\
& $\mathrm{~S}_{3}\left(\mathrm{n}_{\mathrm{O}} \pi^{*}\right)$ & 5.305 & 0.001 & 5.34 & \\
\hline EOM-CCSD/cc-pVDZ & $\mathrm{S}_{0}$ & & & 5.72 & \\
& $\mathrm{~S}_{1}\left(\mathrm{ICT} \pi \pi^{*}\right)$ & 5.032 & 0.700 & 8.45 & -0.187 \\
& $\mathrm{~S}_{2}\left(\mathrm{~L}_{\mathrm{a}} \pi \pi^{*}\right)$ & 5.224 & 0.058 & 6.23 & \\
& $\mathrm{~S}_{3}\left(\mathrm{n}_{\mathrm{O}} \pi^{*}\right)$ & 5.642 & 0.001 & 4.71 & \\
\hline
\end{tabular}

level, at the ground-state equilibrium geometry optimized at the MP2/cc-pVDZ level. The EOM-CCSD calculations were performed within Molpro version 2012.1. ${ }^{5}$ A restricted Hartree-Fock (RHF) reference determinant was used, and the frozen core approximation was applied.

Table S1 compares the vertical excitation spectrum of $8 \mathrm{vG}$ predicted by the MP2/ADC(2) method with that obtained using the EOM-CCSD method. Furthermore, Figure S1 shows the EDDMs for the lowest few singlet electronic excited states of $8 \mathrm{vG}$ calculated using either method. Both methods predict the same energy ordering for the lowest three singlet excited states of $8 \mathrm{vG}$, though the vertical excitation energies calculated using the MP2/ADC $(2)$ method are systematically lower by a few tenths of an electronvolt compared to the EOM-CCSD results.

Regarding the structure of the $\mathrm{S}_{1}$ (ICT $\pi \pi^{*}$ ) state, the EOM-CCSD method confirms the prediction made by the MP2/ADC(2) method that this state involves electron transfer from carbons $\mathrm{C} 4, \mathrm{C} 5$ and $\mathrm{C} 8$ onto atoms N7, N9 and the vinyl group. N1, C2, N3 and N10 are not involved in the $\mathrm{S}_{1}\left(\mathrm{ICT} \pi \pi^{*}\right)$ state to any significant extent, in the sense that the EDDM of this state is not strongly localized on these atoms, and they do not donate or accept significant amounts of charge. Both methods also predict a very similar magnitude and direction of the electric dipole moment of the $\mathrm{S}_{1}\left(\mathrm{ICT} \pi \pi^{*}\right)$ state at the ground-state equilibrium geometry. The only noteworthy - although still relatively minor - point of disagreement between the two methods is the role of carbon C6 and the oxygen atom. According to the MP2/ADC(2) method, the oxygen atom acts as a strong donor of electron density, while carbon C6 is a very weak acceptor. In contrast, the EOM-CCSD method predicts that both these atoms are electron density donors, the oxygen atom being a weak donor and carbon $\mathrm{C} 6$ a strong one. The topological analysis of the EDDMs reveals that both the MP2/ADC(2) and EOM-CCSD methods predict almost the same amount of charge transfer onto the vinyl group upon photoexcitation to the $\mathrm{S}_{1}$ (ICT $\pi \pi^{*}$ ) state: $\Delta q_{\mathrm{v}}=-0.185 e$ according to the MP2/ADC(2) method, and $-0.187 e$ according to the EOM-CCSD method.

What is more, the EOM-CCSD method corroborates the finding that, at the ground-state equilibrium geometry, the $\mathrm{L}_{\mathrm{a}} \pi \pi^{*}$ state of $8 \mathrm{vG}$ lies higher in energy than the ICT $\pi \pi^{*}$ state. As mentioned in the main body of the present paper, this fact is crucial to the fluorescent nature of $8 \mathrm{vG}$, since population of the $\mathrm{L}_{\mathrm{a}} \pi \pi^{*}$ is expected to represent a major radiationless deactivation pathway of $8 \mathrm{vG}$. Both the MP2/ADC $(2)$ and the EOM-CCSD methods predict that the $\mathrm{L}_{\mathrm{a}} \pi \pi^{*}$ state of $8 \mathrm{vG}$ is almost entirely localized on the guanine moiety (and not on the vinyl substituent). The $\mathrm{L}_{\mathrm{a}} \pi \pi^{*}$ state of $8 \mathrm{vG}$ is very similar in structure to the $\mathrm{L}_{\mathrm{a}} \pi \pi^{*}$ state of unmodified guanine, as evidenced by the comparison of the EDDMs of those states (Figures S1 and S3). 
In complete agreement with the EOM-CCSD method, the MP2/ADC (2) combination predicts that the vertical excitation into the lowest $\mathrm{n} \pi^{*}$ state - namely, the $\mathrm{n}_{\mathrm{O}} \pi^{*}$ state mostly localized on the C6-O bond - is well separated in energy (roughly $0.6 \mathrm{eV}$ higher) from the vertical excitation energy into the ICT $\pi \pi^{*}$ state. We may therefore conclude that in the case of $8 \mathrm{vG}$, the use the MP2/ADC(2) combination will not introduce a significant bias towards the $\mathrm{n} \pi^{*}$-type states.

In summary, the EOM-CCSD calculations provide support for the applicability of the MP2/ADC(2) method for the description of the low-lying singlet excited states of $8 \mathrm{vG}$, while at the same time indicating that the MP2/ADC(2) method may provide an erroneous description of the involvement of carbon $\mathrm{C} 6$ and the oxygen atom of $8 \mathrm{vG}$ in the $\mathrm{S}_{1}$ (ICT $\pi \pi^{*}$ ) state. The latter issue seems only of minor importance, however, and on the whole, the two methods are in fairly close agreement as to the structure of the $\mathrm{S}_{1}\left(\mathrm{ICT} \pi \pi^{*}\right)$ state.

Figure S1: (a) EDDMs for the lowest three singlet excited electronic states of $8 \mathrm{vG}$ at its ground-state equilibrium geometry, calculated at the MP2/ADC(2)/cc-pVDZ level of theory, and plotted in the form of isosurfaces with isovalues of $\pm 0.002 e / a_{0}{ }^{3}$. For each excited state, the red and blue isosurfaces delimit regions in which the electron density is increased and decreased, respectively, relative to the $\mathrm{S}_{0}$ state. The ground-state equilibrium geometry was optimized at the MP2/cc-pVDZ level of theory.

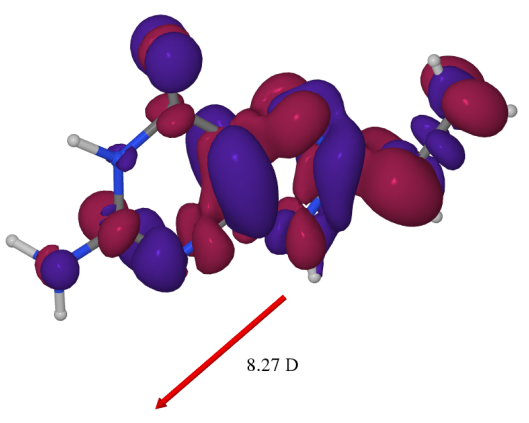

(i) $\mathrm{S}_{1}\left(\mathrm{ICT} \pi \pi^{*}\right)$

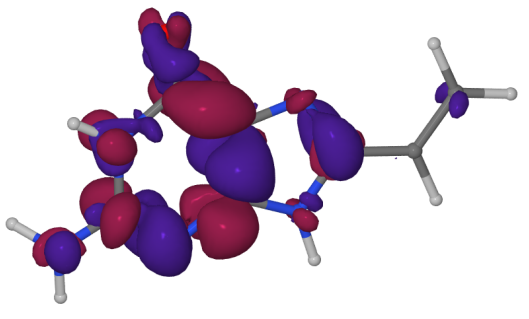

(ii) $\mathrm{S}_{2}\left(\mathrm{~L}_{\mathrm{a}} \pi \pi^{*}\right)$

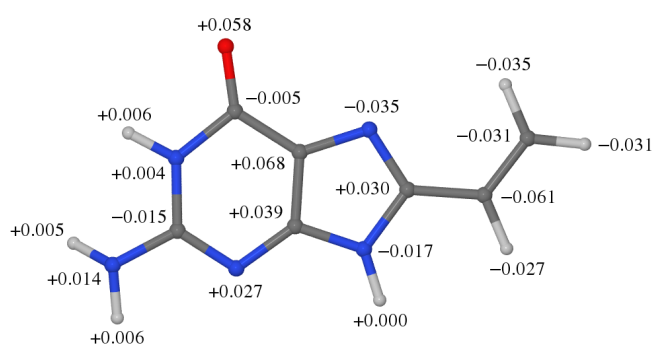

Topological analysis of electron density redistribution in the $\mathrm{S}_{1}\left(\mathrm{ICT} \pi \pi^{*}\right)$ state

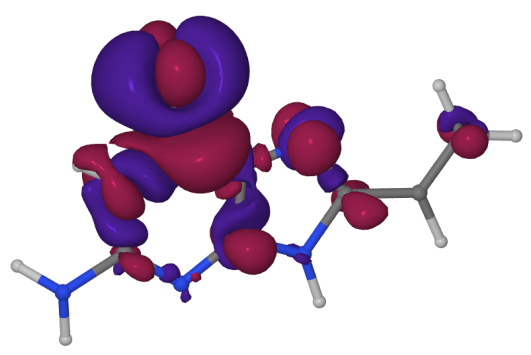


(b) EDDMs for the lowest three singlet excited electronic states of $8 \mathrm{vG}$ at its ground-state equilibrium geometry, calculated at the EOM-CCSD/cc-pVDZ level of theory, and plotted in the form of isosurfaces with isovalues of $\pm 0.002 e / a_{0}{ }^{3}$.

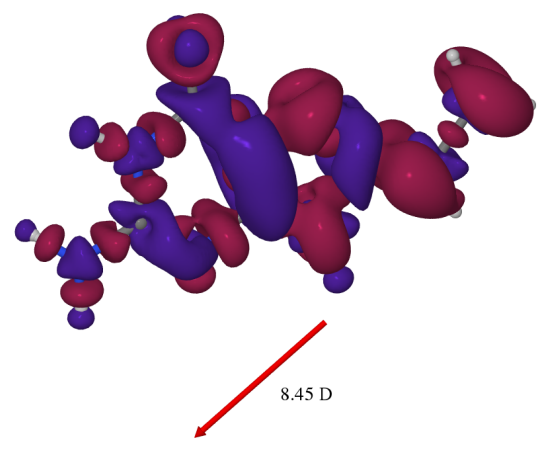

(i) $\mathrm{S}_{1}\left(\mathrm{ICT} \pi \pi^{*}\right)$

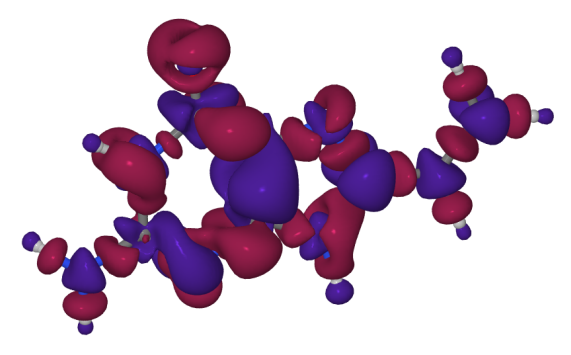

(ii) $\mathrm{S}_{2}\left(\mathrm{~L}_{\mathrm{a}} \pi \pi^{*}\right)$

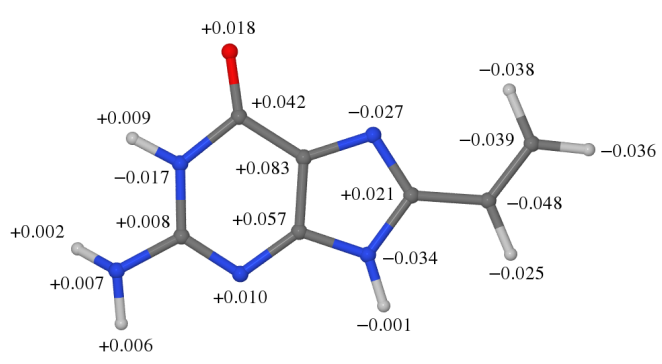

Topological analysis of electron density redistribution in the $\mathrm{S}_{1}$ (ICT $\pi \pi^{*}$ ) state

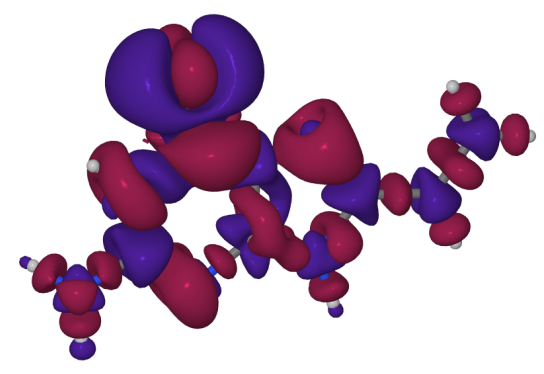

(iii) $\mathrm{S}_{3}\left(\mathrm{n}_{\mathrm{O}} \pi^{*}\right)$ 


\subsection{Potential energy surfaces of $9 \mathrm{H}$-guanine}

\subsubsection{Background}

In this and the following subsections, we demonstrate that the photochemistry of unmodified $9 \mathrm{H}$-guanine predicted by the $\mathrm{MP} 2 / \mathrm{ADC}(2)$ method is in close agreement with benchmarks provided by higher-level electronic structure methods. Our motivation here is twofold. Firstly, from a methodological standpoint, a realistic description of the photochemistry of $9 \mathrm{H}$-guanine by $\mathrm{MP} 2 / \mathrm{ADC}(2)$ is a prerequisite for its application to guanine derivatives. Secondly, the MP2/ADC(2) calculations reported in the main body of the present work indicate that the $\mathrm{L}_{\mathrm{a}} \pi \pi^{*}$ state of $8 \mathrm{vG}$ plays a key role in the non-radiative deactivation of this guanine analogue, which competes with fluorescence emission. Because the $\mathrm{L}_{\mathrm{a}} \pi \pi^{*}$ state of $8 \mathrm{vG}$ is mostly localized on the purine moiety and similar in structure to its counterpart in unmodified $9 \mathrm{H}$-guanine, it can be inferred that the MP2/ADC(2) method also provides a realistic description of the $\mathrm{L}_{\mathrm{a}} \pi \pi^{*}$ state of $8 \mathrm{vG}$.

In order to set the stage of the analysis of the performance of the MP2/ADC(2) method for $9 \mathrm{H}$-guanine, we begin with a brief summary of its photochemistry in the gas-phase. For a more detailed review of previous work on this subject, the Reader is referred to Reference. ${ }^{6}$ Several previous theoretical studies ${ }^{7-15}$ have investigated the relaxation process of $9 \mathrm{H}$-guanine in the gas phase following the irradiation of its lowest absorption band. With the exception of the simulations reported by Langer and Doltsinis, ${ }^{7,12}$ which were based on the restricted open-shell Kohn-Sham method, ${ }^{16}$ these works have largely converged on a uniform description of the relaxation process. Figure S2 shows schematically the predominant relaxation mechanism as established by nonadiabatic molecular dynamics simulations employing semiempirical ${ }^{13}$ and $a b$ initio ${ }^{14,15}$ electronic structure methods.

The initial photoexcitation is into the $\mathrm{S}_{1}\left(\mathrm{~L}_{\mathrm{a}} \pi \pi^{*}\right.$ state) state. (In the Franck-Condon region, the $\mathrm{n}_{\mathrm{O}} \pi^{*}$ state and the lowest $\pi \sigma^{*}$ state are close in energy to the $\mathrm{L}_{\mathrm{a}} \pi \pi^{*}$ state, but the former two states have low oscillator strengths, and are therefore difficult to populate by direct photoexcitation from the ground state.) The majority of the excited-state population subsequently relaxes rapidly on the $\mathrm{S}_{1}\left(\mathrm{~L}_{\mathrm{a}} \pi \pi^{*}\right)$ state along a barrierless downward pathway connecting the Franck-Condon region (meaning, molecular geometries close to the ground state equilibrium geometry) to a conical intersection (CI) seam with the ground state. Molecular geometries at this $\mathrm{L}_{\mathrm{a}} / \pi \pi^{*}$ CI seam are characterized by strong deformations of the six-membered ring, ranging from envelope-type $\left(\mathrm{E}_{2}\right.$ or ${ }^{2} \mathrm{E}$ in the classification of Evans and Boeyens $\left.{ }^{17}\right)$ to boat-type $\left(\mathrm{B}_{2,5}\right.$ or $\left.{ }^{2,5} \mathrm{~B}\right)$, while the five-membered ring remains near-planar. Near the $\mathrm{L}_{\mathrm{a}} / \pi \pi^{*}$ CI seam, the excited-state population undergoes internal conversion to the vibrationally hot electronic ground state. The semiempirical OM2/MRCI simulation study of Lan et al. ${ }^{13}$ predicted that this internal conversion process is characterized by two decay constants of $\tau_{1}=190 \mathrm{fs}$ and $\tau_{2}=400 \mathrm{fs}$, whereas the MRCIS simulations of Barbatti and coworkers $^{15}$ reported decay with a single time constant of $\tau=224 \mathrm{fs}$.

According to the simulation study of Barbatti and coworkers, ${ }^{15}$ a minor fraction (estimated as $5 \%$ ) of the excited-state population does not follow the direct decay pathway on the $\mathrm{S}_{1}\left(\mathrm{~L}_{\mathrm{a}} \pi \pi^{*}\right)$ state, but instead accesses a region of the configuration space where the $\mathrm{S}_{1}$ state has $\mathrm{n}_{\mathrm{O}} \pi^{*}$ character. A potential minimum exists on the $\mathrm{S}_{1}\left(\mathrm{n}_{\mathrm{O}} \pi^{*}\right)$ state, ${ }^{9}$ but the excited-state population is not trapped there for a significant period of time (for at most a few hundred femtoseconds), and instead decays to the ground state via an intersection of the $\mathrm{S}_{1}\left(\mathrm{n}_{\mathrm{O}} \pi^{*}\right)$ state with the ground state.

It is difficult to make a straightforward comparison of simulations of the $9 \mathrm{H}$-guanine and the results of gas-phase spectroscopic experiments, as the experimental data is not tautomer-specific, and the biologically-relevant $9 \mathrm{H}$ tautomer is only a minority tautomer in the gas phase. In the mass-selected pump-probe resonant ionization study of Canuel and coworkers, ${ }^{18}$ the relaxation of guanine photoexcited at a wavelength of $267 \mathrm{~nm}$ was interpreted as a biexponential process with 
Figure S2: Schematic illustration of the relaxation dynamics of UV-excited guanine, adapted from Ref. ${ }^{14}$ Only the main deactivation mechanism, relaxation on the $\mathrm{L}_{\mathrm{a}} \pi \pi^{*}$ state followed by internal conversion to the ground state, is shown in this picture. The reaction coordinate represents relaxation from the Franck-Condon geometry to the $\mathrm{S}_{1}\left(\mathrm{~L}_{\mathrm{a}} \pi \pi^{*}\right) / \mathrm{S}_{0}$ CI seam, or to the minimum on the $\mathrm{S}_{1}\left(\mathrm{n}_{\mathrm{O}} \pi^{*}\right)$ state. The ground-state equilibrium geometry is labelled $\mathrm{S}_{0}$-min, while the CI seam between the $\mathrm{L}_{\mathrm{a}} \pi \pi^{*}$ state and the ground state is marked $\mathrm{L}_{\mathrm{a}} \pi \pi^{*} /$ gs CI. $\tau=224$ fs is the excited-state lifetime estimated from TSH simulations at the MRCIS level. ${ }^{15}$

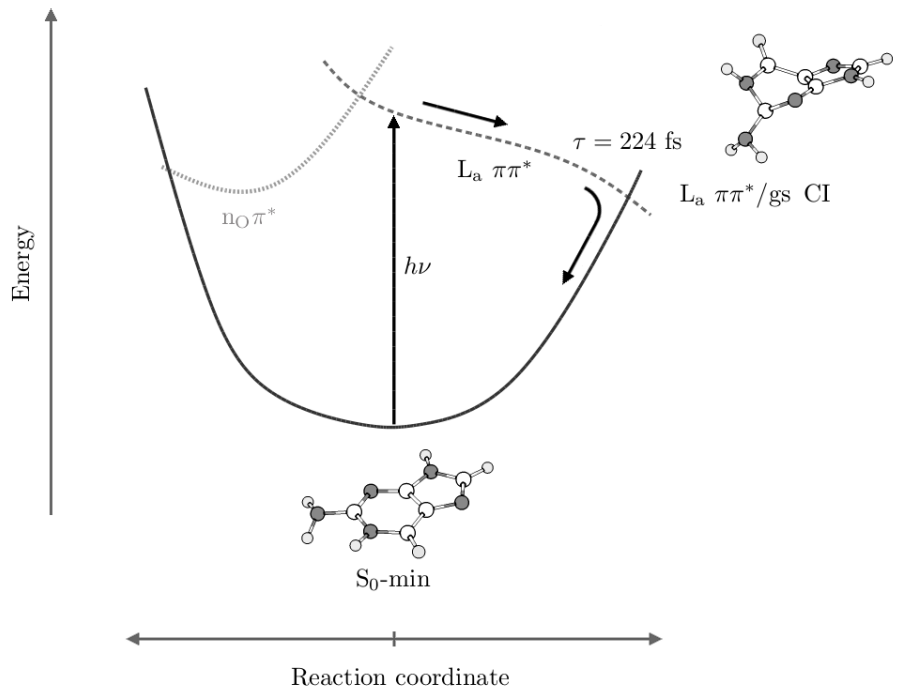

decay constants $\tau_{1}=148$ fs and $\tau_{2}=0.36 \mathrm{ps}$. However, a later re-analysis of the data with the inclusion of all fragmentation channels has suggested that the latter time constant may in fact be considerably longer $(2.3 \mathrm{ps}) .{ }^{19}$ Although at least the shorter of these observed time constants coincides closely with the time constants predicted by simulations, $\left(\tau_{1}=190 \mathrm{fs}\right.$ and $\tau_{2}=400$ fs at the semiempirical OM2/MRCI level, ${ }^{13}$ and $\tau=224$ fs at the MRCIS level ${ }^{15}$ ) it is not known whether either originates from the $9 \mathrm{H}$ tautomer. For these reasons, in our analysis of the performance of the the MP2/ADC(2) method for 9H-guanine, we largely restrict ourselves to benchmarking against other electronic structure methods.

\subsubsection{Computational methods}

Due to the predominant involvement of the direct relaxation pathway on the $\mathrm{L}_{\mathrm{a}} \pi \pi^{*}$ state from the Franck-Condon geometry (i.e. the ground state equilibrium geometry, denoted $\mathrm{S}_{0}$-min) to the $\mathrm{L}_{\mathrm{a}} / \pi \pi^{*}$ CI seam, we first tested the performance of the MP2/ADC(2) method by scanning the potential energy surfaces of guanine along paths in the configuration space of the molecule between $\mathrm{S}_{0}$-min and minima on the crossing seam (MXSs). The method of complete active space second-order perturbation theory (CASPT2) was adopted as a reference against which to assess the accuracy of the MP2/ADC(2) method. Minima on the $\mathrm{S}_{0}$ and $\mathrm{S}_{1}$ potential energy surfaces, and minima on the crossing seam (MXSs) between the $\mathrm{S}_{1}$ and $\mathrm{S}_{0}$ states, were located and optimized at the MP2/ADC(2)/aug-cc-pVDZ level of theory. Subsequently, LIIC paths were generated between the Franck-Condon geometry on one hand, and the $\mathrm{L}_{\mathrm{a}} / \pi \pi^{*}$ MXSs on the other. The ground- and excited-state PESs were scanned along the resulting LIIC paths by means of single-point calculations at both the MP2/ADC(2)/aug-cc-pVDZ and the CASPT2/6-31G(d) levels of theory. 
It should be emphasized that due to its single-reference nature, $\operatorname{MP} 2 / \operatorname{ADC}(2)$ is not capable of providing a formally correct description of structures where the ground state exhibits significant multireference character, such as biradicaloid systems and the vicinity of crossings with the ground state. However, previous experience with the MP2/ADC(2) method indicates that near crossings with the ground state, it exhibits a "graceful failure" behavior, in the sense that it remains numerically stable and the calculated energy gap between the $S_{1}$ and $S_{0}$ states becomes small. ${ }^{20}$ Hence, it can be used to predict the locations of $\mathrm{S}_{1} / \mathrm{S}_{0}$ crossings.

The CASPT2 calculations were performed within Molpro 2012.1 using the single-state implementation of CASPT2. ${ }^{21}$ Two choices of active space were employed. For the purpose of calculating the vertical excitation energies of $9 \mathrm{H}$-guanine at the CASPT2 level, active space I was defined to include the $\mathrm{n}_{\mathrm{O}}$ orbital which corresponds to the oxygen lone electron pair, seven $\pi$-type and four $\pi^{*}$-type orbitals, for a total of 16 electrons distributed in 12 orbitals. Plots of orbitals comprising this active space are shown in Figure S3 (a). In the calculation of the vertical excitation spectrum using active space I, the orbitals were averaged over the lowest four singlet states. We denote calculations performed using active space I as CASSCF-I and CASPT2-I.

In order to provide a benchmark for assessing the accuracy of the MP2/ADC(2) method for the PES of the $\mathrm{L}_{\mathrm{a}} \pi \pi^{*}$ state, it is sufficient to include in the CASPT2 calculation the singlet ground state and the $\mathrm{L}_{\mathrm{a}} \pi \pi^{*}$ state, but not the $\mathrm{n}_{\mathrm{O}} \pi^{*}$ and the $\mathrm{L}_{\mathrm{b}} \pi \pi^{*}$ states. Therefore, a in the PES scans a different choice of active space was used, which included seven $\pi$-type and four $\pi^{*}$-type orbitals. Thus, active space II correlated 14 electrons in 11 orbitals. With active space II, the orbitals were averaged over only two adiabatic states. The use of active space II is denoted as CASSCF-II and CASPT2-II.

The 6-31G(d) basis set was employed in all CASSCF and CASPT2 calculations. We found it necessary to apply an IPEA shift ${ }^{22}$ of 0.25 a.u. in order to avoid intruder state problems. Because of the use of a highly localized basis set and exclusion of Rydberg-type orbitals from either active space, the diffuse Rydberg-type excited states of 9H-guanine are not detected in the CASSCF and CASPT2 calculations.

\subsubsection{Equilibrium geometries and MXSs of 9H-guanine}

Figure S4 shows the relevant geometries of 9H-guanine as optimized at the MP2/ADC(2)/aug-cc-pVDZ level of theory. The conformation of the six-membered ring in each of these structures has been characterized using the scheme of Hill and Reilly, ${ }^{23}$ whereby the puckering of a six-membered ring is described by means of a set of three angles $\theta_{0}, \theta_{1}$ and $\theta_{2}$ (where $-90^{\circ} \leq \theta_{i} \leq 90^{\circ}$ ). The relative energies and conformations of each structure are listed in Table S2. 
Figure S3 (a) Natural orbitals comprising active space I, at the ground-state equilibrium geometry of $9 \mathrm{H}$-guanine, plotted in the form of isosurfaces with isovalues of $\pm 0.05 a_{0}{ }^{-3}$. The ground-state geometry was optimized at the MP2/ADC(2)/aug-cc-pVDZ level of theory.

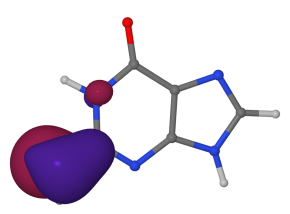

$\pi_{1}$
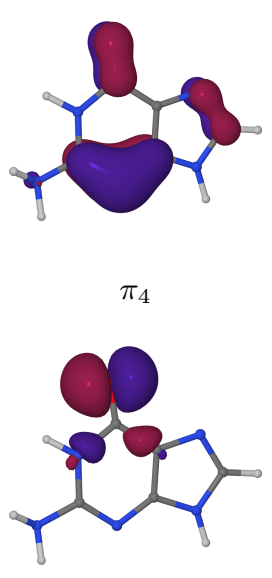

no

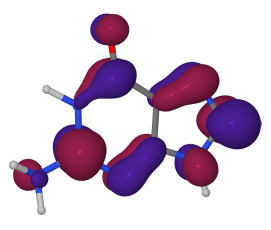

$\pi_{2}^{*}$

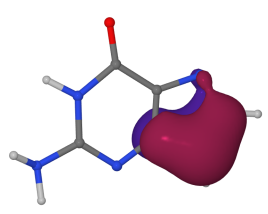

$\pi_{2}$

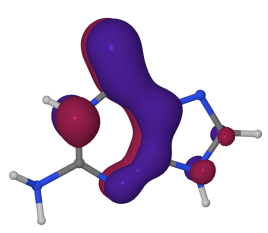

$\pi_{5}$

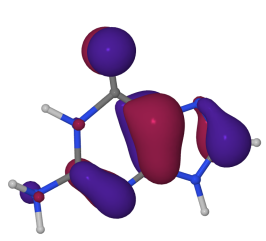

$\pi_{7}$

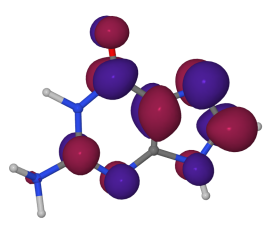

$\pi_{3}^{*}$

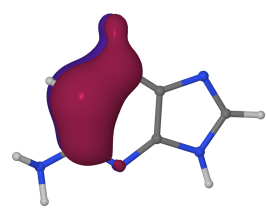

$\pi_{3}$

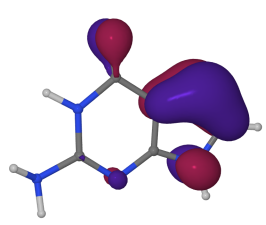

$\pi_{6}$

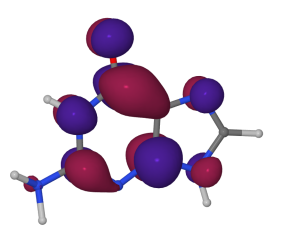

$\pi_{1}^{*}$

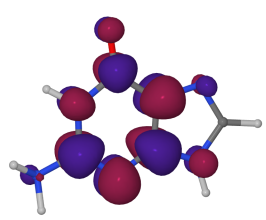

$\pi_{4}^{*}$ 
(b) Natural orbitals comprising active space II.

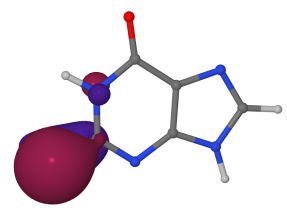

$\pi_{1}$

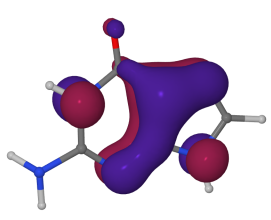

$\pi_{4}$

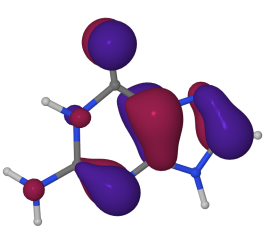

$\pi_{7}$

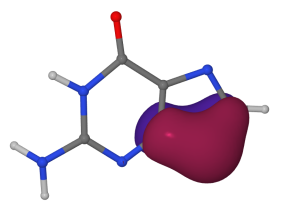

$\pi_{2}$

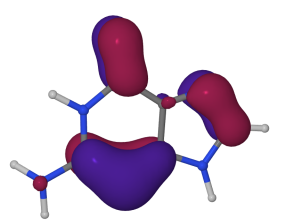

$\pi_{5}$

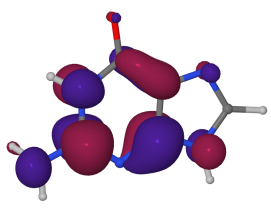

$\pi_{1}^{*}$

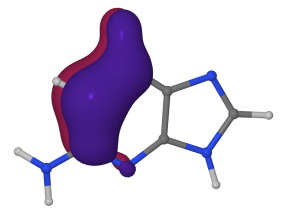

$\pi_{3}$

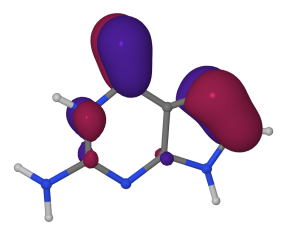

$\pi_{6}$

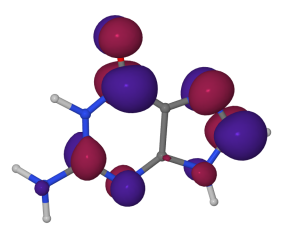

$\pi_{2}^{*}$

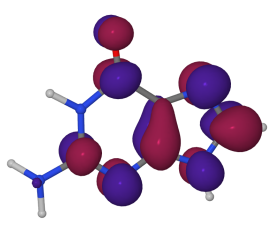

$\pi_{3}^{*}$

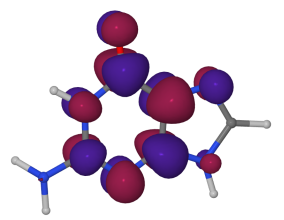

$\pi_{4}^{*}$

The ground-state equilibrium geometry $\left(\mathrm{S}_{0}-\mathrm{min}\right)$ of $9 \mathrm{H}$-guanine is near-planar, except that the amino nitrogen N10 is slightly pyramidalized. The vertical excitation spectra at $\mathrm{S}_{0}$-min, calculated at the MP2/ADC(2)/aug-cc-pVDZ and CASSCF/6-31G(d) and CASPT2/6-31G(d) levels of theory, using either choice of active space, are analyzed in Table S3. Furthermore, Figure S5 shows EDDMs for the singlet excited electronic states at $\mathrm{S}_{0}$-min, calculated at the MP2/ADC(2)/aug-cc-pVDZ level as well as at the CASSCF/6-31G(d) level. According to both the MP2/ADC(2)/aug-cc-pVDZ and CASPT2-I/6-31G(d) levels of theory, the lowest vertical excitation is into the $\mathrm{L}_{\mathrm{a}} \pi \pi^{*}$ state, which is closely followed by the $\mathrm{n}_{\mathrm{O}} \pi^{*}$ state. In turn, the $\mathrm{L}_{\mathrm{b}} \pi \pi^{*}$ state lies above the $\mathrm{n}_{\mathrm{O}} \pi^{*}$ state. The same energy ordering of the $\mathrm{L}_{\mathrm{a}} \pi \pi^{*}, \mathrm{n}_{\mathrm{O}} \pi^{*}$ and $\mathrm{L}_{\mathrm{b}} \pi \pi^{*}$ states was reported in some previous simulation studies using the CASPT2 method, ${ }^{9,10}$ with the exception of the work of Chen and $\mathrm{Li}^{8}{ }^{8}$ where the $\mathrm{n}_{\mathrm{O}} \pi^{*}$ state was reported to lie around $0.1 \mathrm{eV}$ above the $\mathrm{L}_{\mathrm{b}} \pi \pi^{*}$ state. High-level coupled cluster calculations $^{2}$ also place $\mathrm{n}_{\mathrm{O}} \pi^{*}$ slightly above the $\mathrm{L}_{\mathrm{b}} \pi \pi^{*}$ state state, meaning that at present, it is not possible to conclusively determine the energy ordering of these two states. The vertical excitation energies calculated at the MP2/ADC(2)/aug-cc-pVDZ level are systematically lower by a few tenths of an electronvolt than the corresponding values from the CASPT2-I and CASPT2-II calculations, 
Table S2: Characterization of relevant geometries on the ground- and excited-state PESs of $9 \mathrm{H}$-guanine as optimized at the MP2/ADC(2)/aug-cc-pVDZ level of theory. The column labeled 'State' identifies the electronic state(s) on which a given structure exists. Energy values are quoted relative to the $\mathrm{S}_{0}$-min structure, and do not include zero-point vibrational corrections. The conformation of the six-membered ring is assigned using the Hill-Reilly algorithm ${ }^{23}$ and labeled by the convention of Evans and Boeyens. ${ }^{17}$

\begin{tabular}{|l|l|l|l|l|l|l|}
\hline Structure & State & $E, \mathrm{eV}$ & $\theta_{0}$, & $\theta_{1}$ & $\theta_{2}$ & Conformation \\
\hline $\mathrm{S}_{0}-\mathrm{min}$ & $\mathrm{S}_{0}$ & 0 & $0.02^{\circ}$ & $-1.71^{\circ}$ & $0.57^{\circ}$ & near-planar \\
$\mathrm{S}_{1}-\mathrm{n}_{\mathrm{O}} \pi^{*}$-min & $\mathrm{S}_{1}\left(\mathrm{n}_{\mathrm{O}} \pi^{*}\right)$ & 3.942 & $4.17^{\circ}$ & $-3.03^{\circ}$ & $14.20^{\circ}$ & ${ }^{6} \mathrm{E}$ \\
MXS1 & $\mathrm{S}_{1}\left(\mathrm{~L}_{\mathrm{a}} \pi \pi^{*}\right) / \mathrm{S}_{0}$ & 3.694 & $71.97^{\circ}$ & $-15.88^{\circ}$ & $-22.16^{\circ}$ & ${ }_{2,5} \mathrm{~B}$ \\
MXS2 & $\mathrm{S}_{1}\left(\mathrm{~L}_{\mathrm{a}} \pi \pi^{*}\right) / \mathrm{S}_{0}$ & 3.718 & $77.14^{\circ}$ & $-19.66^{\circ}$ & $-5.98^{\circ}$ & ${ }^{2,5} \mathrm{~B}$ \\
MXS3 & $\mathrm{S}_{1}\left(\mathrm{~L}_{\mathrm{a}} \pi \pi^{*}\right) / \mathrm{S}_{0}$ & 3.348 & $50.59^{\circ}$ & $-5.92^{\circ}$ & $-2.50^{\circ}$ & ${ }^{2} \mathrm{E}$ \\
MXS4 & $\mathrm{S}_{1}\left(\mathrm{n}_{\mathrm{O}} \pi^{*}\right) / \mathrm{S}_{0}$ & 4.061 & $-23.52^{\circ}$ & $14.77^{\circ}$ & $-38.79^{\circ}$ & $\mathrm{E}_{6}$ \\
MXS5 & $\mathrm{S}_{1}\left(\mathrm{n}_{\mathrm{O}} \pi^{*}\right) / \mathrm{S}_{0}$ & 4.551 & $-1.40^{\circ}$ & $10.00^{\circ}$ & $-37.95^{\circ}$ & $\mathrm{E}_{6}$ \\
\hline
\end{tabular}

Table S3: Vertical excitation energies $(\Delta E)$, oscillator strengths $(f)$ and electric dipole moments $(\mu)$ of $9 \mathrm{H}$-guanine at its ground-state equilibrium geometry, calculated at the MP2/ADC(2)/aug-cc-pVDZ, CASSCF/6-31G(d) and CASPT2/6-31G(d) levels of theory. The calculation of oscillator strengths and electric dipole moments is not available for the CASPT2 method.

\begin{tabular}{|l|l|l|l|l|}
\hline Level of theory & State & $\Delta E, \mathrm{eV}$ & $f$ & $\mu, \mathrm{D}$ \\
\hline MP2/ADC(2)/aug-cc-pVDZ & $\mathrm{S}_{0}$ & & & 6.30 \\
& $\mathrm{~S}_{1}\left(\mathrm{~L}_{\mathrm{a}} \pi \pi^{*}\right)$ & 4.841 & 0.153 & 5.48 \\
& $\mathrm{~S}_{2}\left(\pi \sigma^{*}\right)$ & 4.952 & 0.020 & 4.32 \\
& $\mathrm{~S}_{3}\left(\mathrm{n}_{\mathrm{O}} \pi^{*}\right)$ & 5.113 & 0.001 & 4.35 \\
& $\mathrm{~S}_{4}\left(\pi \sigma^{*}\right)$ & 5.294 & 0.047 & 6.02 \\
& $\mathrm{~S}_{5}\left(\mathrm{~L}_{\mathrm{b}} \pi \pi^{*}\right)$ & 5.338 & 0.280 & 5.97 \\
& $\mathrm{~S}_{6}\left(\pi \sigma^{*}\right)$ & 5.900 & 0.004 & 6.97 \\
\hline CASSCF-I/6-31G(d) & $\mathrm{S}_{0}$ & & & 5.93 \\
& $\mathrm{~S}_{1}\left(\mathrm{n}_{\mathrm{O}} \pi^{*}\right)$ & 5.364 & 0.001 & 3.72 \\
& $\mathrm{~S}_{2}\left(\mathrm{~L}_{\mathrm{a}} \pi \pi^{*}\right)$ & 5.657 & 0.207 & 5.45 \\
& $\mathrm{~S}_{3}\left(\mathrm{~L}_{\mathrm{b}} \pi \pi^{*}\right)$ & 6.550 & 0.090 & 5.20 \\
\hline CASPT2-I/6-31G(d) & $\mathrm{S}_{0}$ & & & \\
& $\mathrm{~S}_{1}\left(\mathrm{~L}_{\mathrm{a}} \pi \pi^{*}\right)$ & 5.112 & & \\
& $\mathrm{~S}_{2}\left(\mathrm{n}_{\mathrm{O}} \pi^{*}\right)$ & 5.595 & & \\
& $\mathrm{~S}_{3}\left(\mathrm{~L}_{\mathrm{b}} \pi \pi^{*}\right)$ & 6.053 & & \\
\hline CASSCF-II/6-31G(d) & $\mathrm{S}_{0}$ & & & 6.02 \\
& $\mathrm{~S}_{1}\left(\mathrm{~L}_{\mathrm{a}} \pi \pi^{*}\right)$ & 5.660 & 0.250 & 5.27 \\
\hline CASPT2-II/6-31G(d) & $\mathrm{S}_{0}$ & & & \\
& $\mathrm{~S}_{1}\left(\mathrm{~L}_{\mathrm{a}} \pi \pi^{*}\right)$ & 5.118 & & \\
\hline
\end{tabular}

which is partially attributable to the fact that a larger basis set was used with the MP2/ADC $(2)$ method than in the latter calculations.

As seen in Figure S5, at both the MP2/ADC(2)/aug-cc-pVDZ and CASSCF-I/6-31G(d) levels of theory, the EDDM for the $\mathrm{L}_{\mathrm{a}} \pi \pi^{*}$ state is mainly localized along the $\mathrm{C} 2-\mathrm{N} 3-\mathrm{C} 4-\mathrm{C} 5$ region of the six-membered ring. This is consistent with the observation made by $\mathrm{Chen}$ and $\mathrm{Li}^{8}$ that the $\mathrm{L}_{\mathrm{a}} \pi \pi^{*}$ state is predominantly a $\mathrm{HOMO} \rightarrow$ LUMO excitation, with the HOMO being localized largely on $\mathrm{N} 3$ and $\mathrm{C} 5$, and the LUMO mainly on $\mathrm{C} 2$ and $\mathrm{C} 4$. 
Due to the near-lack of dynamical correlation in the CASSCF-I/6-31G(d) calculation, at this level of theory the energetic ordering of the $\mathrm{L}_{\mathrm{a}} \pi \pi^{*}$ state and $\mathrm{n}_{\mathrm{O}} \pi^{*}$ states is inverted relative to MP2/ADC(2)/aug-cc-pVDZ, CASPT2-I/6-31G(d) and CASPT2-II/6-31G(d), and furthermore all vertical excitation energies are overestimated. Also, for unknown reasons, the MP2/ADC(2)/aug-cc-pVDZ calculation predicts a considerably higher value for the oscillator strength of excitation into the $\mathrm{L}_{\mathrm{b}} \pi \pi^{*}$ state $(0.280)$ than the CASSCF-I/6-31G(d) calculation (0.090). Given that the $\mathrm{L}_{\mathrm{b}} \pi \pi^{*}$ state is not involved in the relaxation dynamics of guanine following the irradiation of its lowest absorption band, we did not investigate this issue further. The vertical excitation energies into the $S_{1}\left(L_{a} \pi \pi^{*}\right)$ state calculated with both choices of active space are very close to one another.

No minimum could be located on the $\mathrm{S}_{1}\left(\mathrm{~L}_{\mathrm{a}} \pi \pi^{*}\right)$ PES of $9 \mathrm{H}$-guanine; all attempts to optimize such a structure resulted in the geometry optimization procedure relaxing to the CI seam between the $\mathrm{L}_{\mathrm{a}} \pi \pi^{*}$ state and the ground state. A single minimum, which we label $\mathrm{S}_{1}-\mathrm{n} \pi^{*}$-min, was found in the region where the $\mathrm{S}_{1}$ state has $\mathrm{n}_{\mathrm{O}} \pi^{*}$ character. Its structure, shown in Figure $\mathrm{S} 4$ (b), is characterized by a slight envelope-type $\left({ }^{6} \mathrm{E}\right)$ deformation of the six-membered ring, and by the elongation of the C6-O bond to 1.432 Å.

A total of three minima on the $\mathrm{L}_{\mathrm{a}} \pi \pi^{*} / \mathrm{gs}$ CI seam were located; we label those MXS1, MXS2 and MXS3. Previous studies which employed the CASSCF ${ }^{8,9}$ and MR-CIS ${ }^{15}$ methods in geometry optimizations, have reported similar geometries of minima along the $\mathrm{L}_{\mathrm{a}} \pi \pi^{*} /$ gs CI seam. The energies of all three of these MXSs lie well below the energy of the $\mathrm{S}_{1}\left(\mathrm{~L}_{\mathrm{a}} \pi \pi^{*}\right)$ state at $\mathrm{S}_{0}-\mathrm{min}$ $(1.147 \mathrm{eV}, 1.123 \mathrm{eV}$ and $1.493 \mathrm{eV}$ in the case of MXS1, MXS2 and MXS3, respectively), which is again consistent with the findings of the works just cited.

MXS1 and MXS2 are close to one another in terms of structure, differing mainly by the position of the hydrogen atom bonded to nitrogen N1. Both exhibit a pronounced boat-type $\left({ }^{2,5} \mathrm{~B}\right)$ deformation of the six-membered ring, an elongation of the $\mathrm{C} 2-\mathrm{N} 3$ and $\mathrm{C} 4-\mathrm{C} 5$ bonds, and a shortening of the N3-C4 bond, in comparison to the $\mathrm{S}_{0}$-min structure. The third minimum on the $\mathrm{L}_{\mathrm{a}} \pi \pi^{*} /$ gs CI seam, MXS3, is characterized by an envelope-type $\left({ }^{2} \mathrm{E}\right)$ deformation of the six-membered ring, a strong displacement of the amino group away from the plane of the ring, as well as an elongation of the $\mathrm{C} 2-\mathrm{N} 3$ and $\mathrm{C} 4-\mathrm{C} 5$ bonds and a shortening of the N3-C4 bond.

Moreover, two minima on the $S_{1} / S_{0}$ CI seam were located where the $S_{1}$ state has $n_{O} \pi^{*}$ character; these are MXS4 and MXS5. In MXS4, atom C6 along with the adjacent oxygen are strongly displaced from the plane of the six-membered ring, such that the N1-C6 bond is elongated to $1.979 \AA$. Essentially the same minimum geometry along the $\mathrm{n}_{\mathrm{O}} \pi^{*} / \mathrm{gs}$ CI seam was reported in previous studies. ${ }^{9,15}$

Finally, MXS5 shows an envelope-type $\left(\mathrm{E}_{6}\right)$ distortion of the six-membered ring, and an elongation of the $\mathrm{C} 6-\mathrm{O}$ bond to $1.573 \AA$. The hydrogen atom bonded to nitrogen N1 is displaced away from the plane of the ring. It seems that no similar minimum along the $\mathrm{S}_{1} / \mathrm{S}_{0}$ CI seam with a displaced $\mathrm{N} 1-\mathrm{H}$ bond has been reported in previous studies; in the present work, the existence of MXS5 was discovered during the analysis of the results of the trajectory surface hopping simulations of the relaxation dynamics of $9 \mathrm{H}$-guanine (see Subsection 1.3). MXS5 was seen to be approached, but not reached, in two of the simulated trajectories, which prompted us to locate and optimize its geometry. It lies relatively high in energy (only $0.290 \mathrm{eV}$ below the energy of the $\mathrm{S}_{1}\left(\mathrm{~L}_{\mathrm{a}} \pi \pi^{*}\right.$ ) state at $\mathrm{S}_{0}$-min), which may explain why no simulated trajectory deactivated in the vicinity of MXS5. 
Figure S4: Relevant geometries of guanine as optimized at the MP2/ADC(2)/aug-cc-pVDZ level of theory. Selected bond lengths are marked in units of $\AA$.

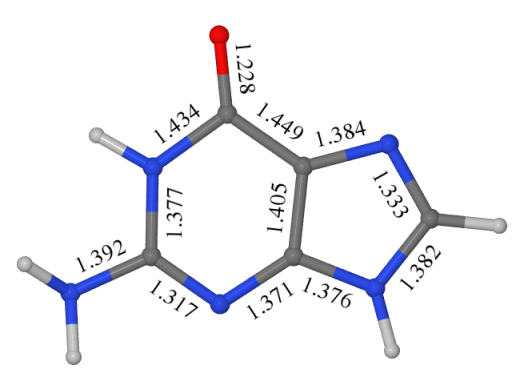

(a) $\mathrm{S}_{0}-\mathrm{min}$
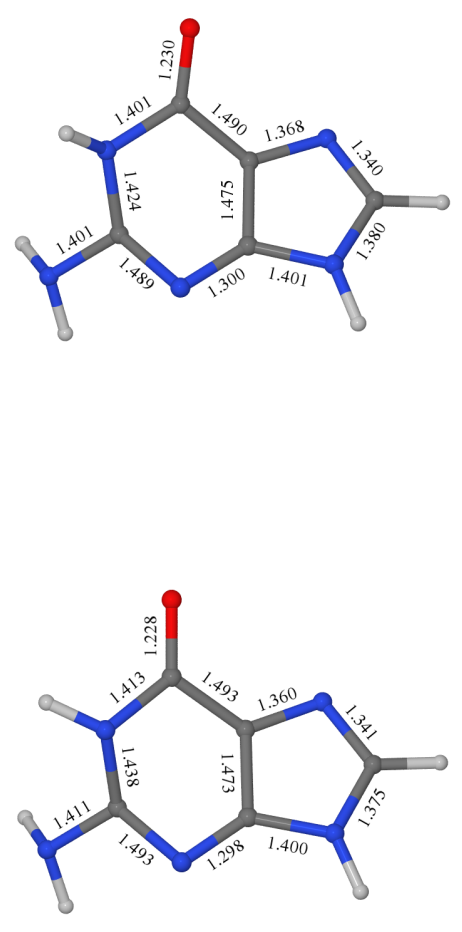

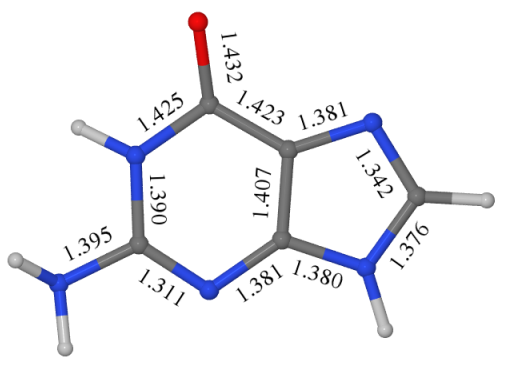

(b) $\mathrm{S}_{1}-\mathrm{n} \pi^{*}-\min$

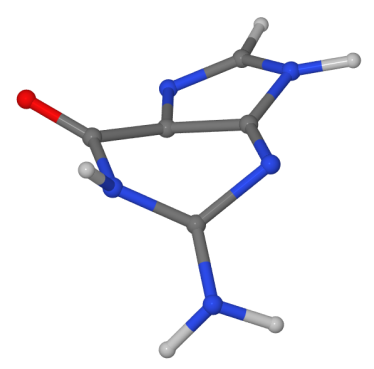

(c) MXS1

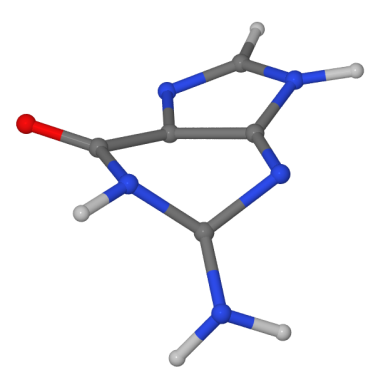

(d) MXS2 

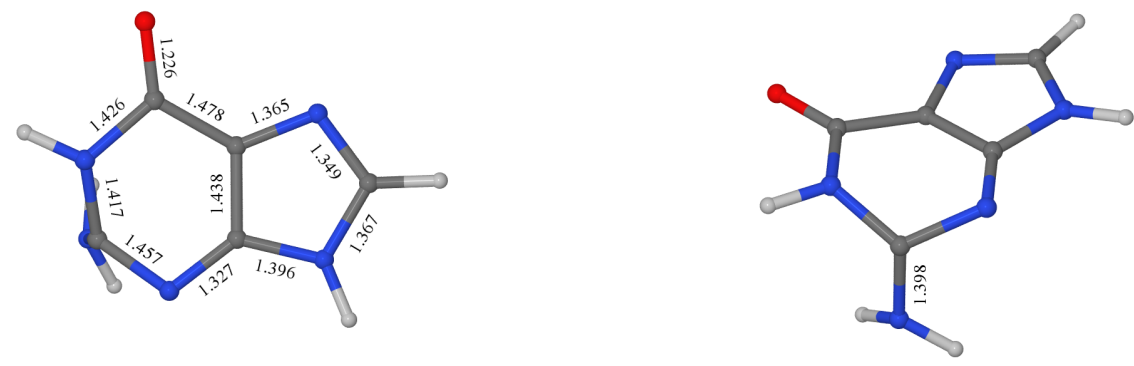

(e) MXS3
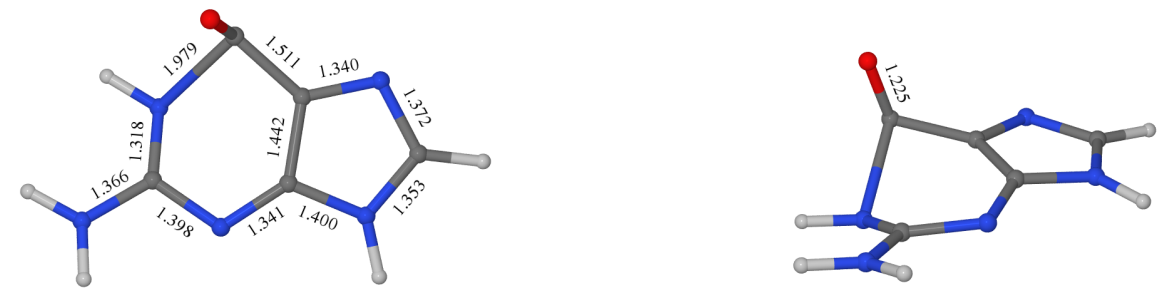

(f) MXS4
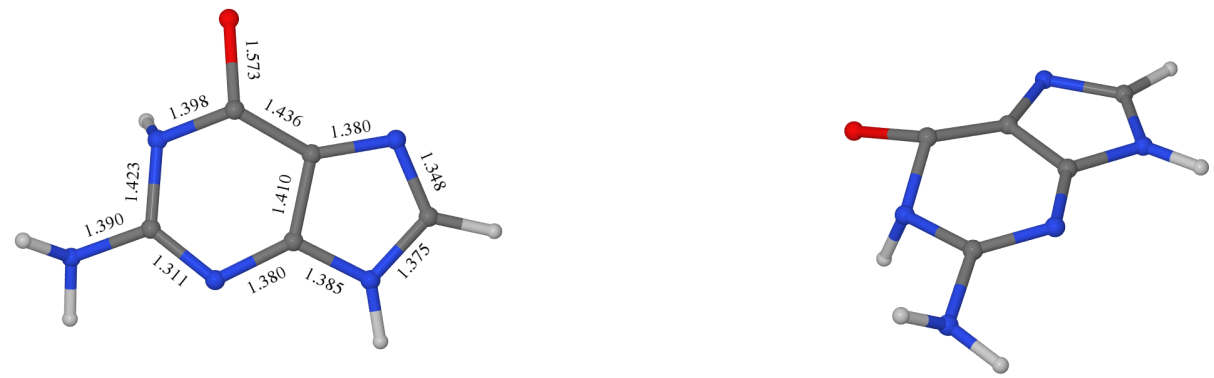

(g) MXS5 
Figure S5: (a) EDDMs for the six lowest singlet excited states of $\mathrm{G}$ at its ground-state equilibrium geometry as calculated at the MP2/ADC(2)/aug-cc-pVDZ level of theory. The EDDMs are plotted in the form of isosurfaces with isovalues of $\pm 0.002 e / a_{0}{ }^{3}$. For each excited state, the red and blue isosurfaces delimit regions in which the electron density is increased and decreased, respectively, relative to the $\mathrm{S}_{0}$ state.

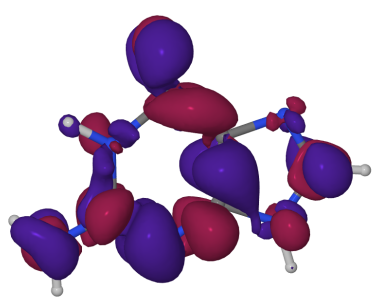

(i) $\mathrm{S}_{1}\left(\mathrm{~L}_{\mathrm{a}} \pi \pi^{*}\right)$

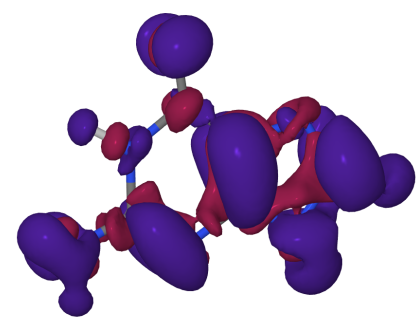

(iv) $\mathrm{S}_{4}\left(\pi \sigma^{*}\right)$

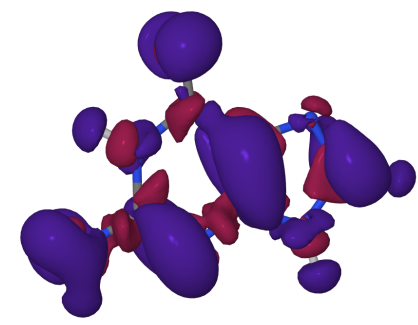

(ii) $\mathrm{S}_{2}\left(\pi \sigma^{*}\right)$

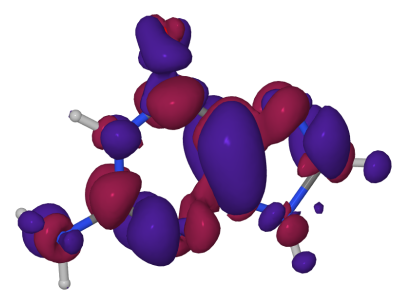

(v) $\mathrm{S}_{5}\left(\mathrm{~L}_{\mathrm{b}} \pi \pi^{*}\right)$

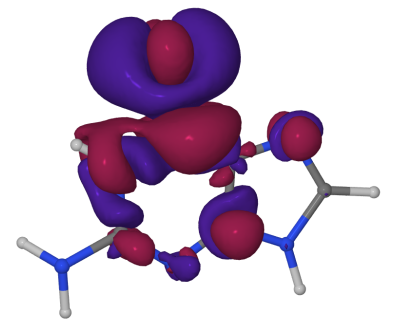

(iii) $\mathrm{S}_{3}\left(\mathrm{n}_{\mathrm{O}} \pi^{*}\right)$

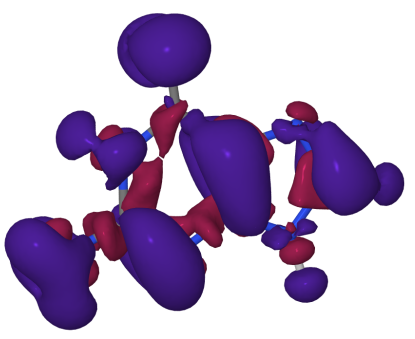

(vi) $\mathrm{S}_{6}\left(\pi \sigma^{*}\right)$

(b) EDDMs for the three lowest singlet excited states of G at the same geometry, calculated at the CASSCF-I/6-31G(d) level of theory. Note that at the CASSCF-I/6-31G(d) level, the energetic ordering of the $\mathrm{n}_{\mathrm{O}} \pi^{*}$ and $\mathrm{L}_{\mathrm{a}} \pi \pi^{*}$ states is inverted relative to the CASPT2-I/6-31G(d) calculation.

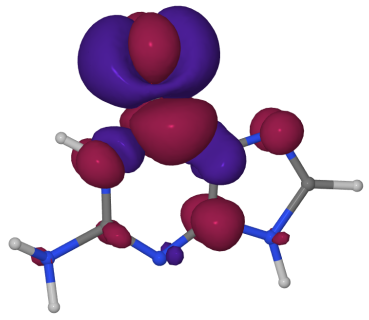

(i) $\mathrm{S}_{1}\left(\mathrm{n}_{\mathrm{O}} \pi^{*}\right)$

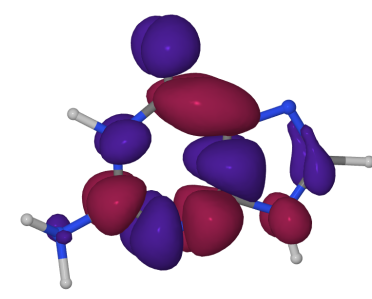

(ii) $\mathrm{S}_{2}\left(\mathrm{~L}_{\mathrm{a}} \pi \pi^{*}\right)$

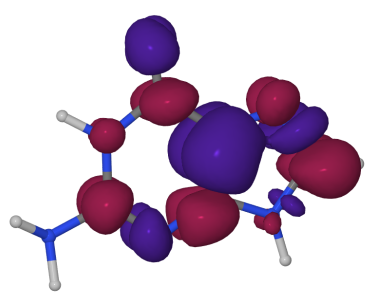

(iii) $\mathrm{S}_{3}\left(\mathrm{~L}_{\mathrm{b}} \pi \pi^{*}\right)$ 


\subsubsection{Potential energy surface scans}

Figure S6 summarizes the results of the MP2/ADC(2)/aug-cc-pVDZ and CASPT2-II/6-31G(d) PES scans along LIIC paths between the $\mathrm{S}_{0}$-min structure of $9 \mathrm{H}$-guanine, and MXS1, MXS2, and MXS3. We discuss first the results of calculations performed with the MP2/ADC(2) method, and then compare them to the benchmark provided by the CASPT2-II calculations.

Inspection of Figure S6 (a), where the results of the MP2/ADC(2)/aug-cc-pVDZ scans are shown, reveals that the PES of the $\mathrm{L}_{\mathrm{a}} \pi \pi^{*}$ state in the Franck-Condon region is steeply sloped towards the $\mathrm{L}_{\mathrm{a}} \pi \pi^{*} / \mathrm{gs}$ CI seam. Along the LIIC paths from $\mathrm{S}_{0}$-min to MXS1, and from $\mathrm{S}_{0}$-min to MXS2, the potential energy of the $\mathrm{S}_{1}\left(\mathrm{~L}_{\mathrm{a}} \pi \pi^{*}\right)$ state decreases sharply before going through very shallow minimuma just before the $\mathrm{L}_{\mathrm{a}} \pi \pi^{*} / \mathrm{gs}$ CI seam. (These apparent minima do not correspond to minima on the fully-dimensional PES of the $\mathrm{S}_{1}$ state; they are merely minima along the LIIC path, which is a one-dimensional cross-section of the PES.) No minimum appears on the LIIC path from $\mathrm{S}_{0}$-min to MXS3; the energy of the $\mathrm{S}_{1}\left(\mathrm{~L}_{\mathrm{a}} \pi \pi^{*}\right)$ state decreases monotonically along this entire path. The steep gradient on the $\mathrm{L}_{\mathrm{a}} \pi \pi^{*}$ state in the Franck-Condon region means that molecules excited into the $\mathrm{L}_{\mathrm{a}} \pi \pi^{*}$ state will be driven along the direct diabatic pathway on this state to the $\mathrm{L}_{\mathrm{a}} \pi \pi^{*} / \mathrm{gs}$ CI seam. The energies of all other low-lying excited electronic states of $9 \mathrm{H}$-guanine increase along the LIIC paths from $\mathrm{S}_{0}$-min to MXS1, MXS2 and MXS3, which suggests that once a molecule leaves the Franck-Condon region while in the $\mathrm{L}_{\mathrm{a}} \pi \pi^{*}$ state, population transfer to another state is unlikely. The above observations are consistent with the fairly homogeneous photorelaxation mechanism predicted by previous nonadiabatic molecular dynamics simulations. ${ }^{13,15}$

Figure $\mathrm{S} 6(\mathrm{~b})$ compares the energies of the $\mathrm{S}_{0}$ and $\mathrm{S}_{1}\left(\mathrm{~L}_{\mathrm{a}} \pi \pi^{*}\right)$ states of $9 \mathrm{H}$-guanine along the LIIC paths from $\mathrm{S}_{0}$-min to MXS1, MXS2 and MXS3, calculated at the MP2/ADC(2)/aug-cc-pVDZ and CASPT2-II/6-31G(d) levels of theory. The energy gap between the $\mathrm{S}_{1}\left(\mathrm{~L}_{\mathrm{a}} \pi \pi^{*}\right)$ and $\mathrm{S}_{0}$ states along each of the three LIIC paths is consistently higher at the CASPT2-II/6-31G(d) level than at the MP2/ADC(2)/aug-cc-pVDZ level; this can partially be ascribed to the fact that a more flexible basis set was used in the MP2/ADC $(2)$ calculation. In other respects, the potential energy curves calculated using the MP2/ADC(2) method are in close agreement with the CASPT2-II benchmark. At each of the three MXS geometries optimized at the MP2/ADC(2)/aug-cc-pVDZ level, the energy gap between the $\mathrm{S}_{1}$ and $\mathrm{S}_{0}$ states computed at the CASPT2-II level is small (0.193 eV at MXS1, $0.162 \mathrm{eV}$ at MXS2, and $0.362 \mathrm{eV}$ at MXS3), indicating that the MP2/ADC(2) method correctly predicts the location of the $\mathrm{L}_{\mathrm{a}} \pi \pi^{*} / \mathrm{gs}$ CI seam.

In summary, we have demonstrated that the MP2/ADC(2) method provides an accurate description of the $\mathrm{L}_{\mathrm{a}} \pi \pi^{*}$ state of unmodified $9 \mathrm{H}$-guanine; this conclusion can be extrapolated to the very similar $\mathrm{L}_{\mathrm{a}} \pi \pi^{*}$ state of $8 \mathrm{vG}$. In fact, the close agreement of the MP2/ADC(2) method with the CASPT2 benchmark is encouraging enough that we have decided to further test the former method's performance by applying it in dynamical (time-resolved) simulations of the relaxation of $9 \mathrm{H}$-guanine photoexcited in the UV range. These simulations are described over the following subsection. 
Figure S6: (a) MP2/ADC(2)/aug-cc-pVDZ energies of singlet electronic states of 9H-guanine along LIIC paths from the Franck-Condon geometry $\left(\mathrm{S}_{0}-\mathrm{min}\right)$ to MXS1 and MXS2. The origin of the mass-weighted distance coordinate corresponds to the $\mathrm{S}_{0}$-min geometry, and the zero of the energy scale corresponds to the energy of the $\mathrm{S}_{0}$ state at $\mathrm{S}_{0}$-min.
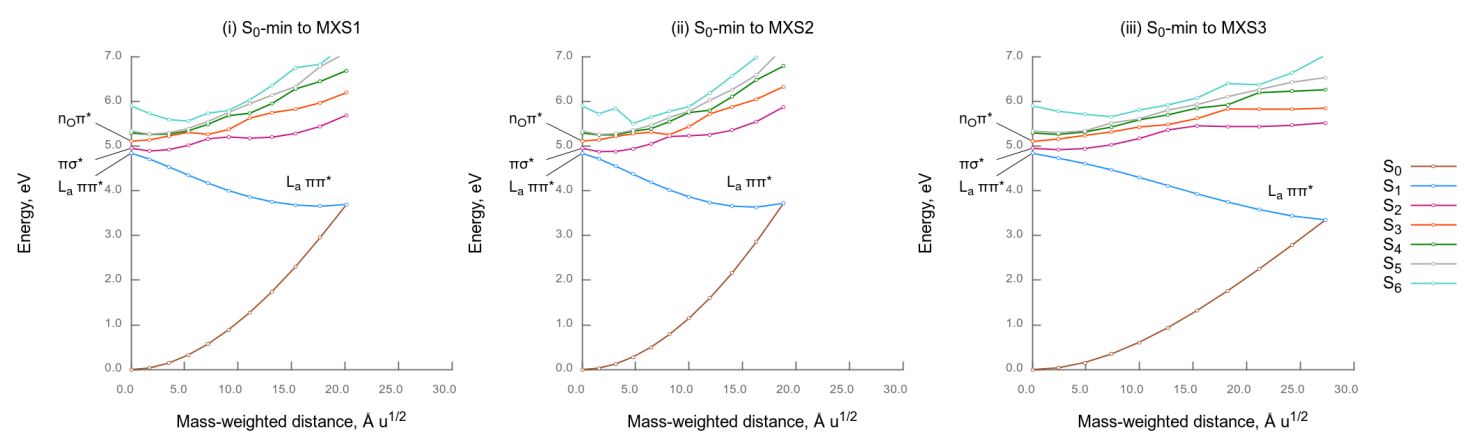

(b) Comparison of the energies of the $\mathrm{S}_{1}\left(\mathrm{~L}_{\mathrm{a}} \pi \pi^{*}\right)$ state and the ground state $\left(\mathrm{S}_{0}\right)$ from the MP2/ADC(2)/aug-cc-pVDZ and CASPT2-II/6-31G(d) calculations.
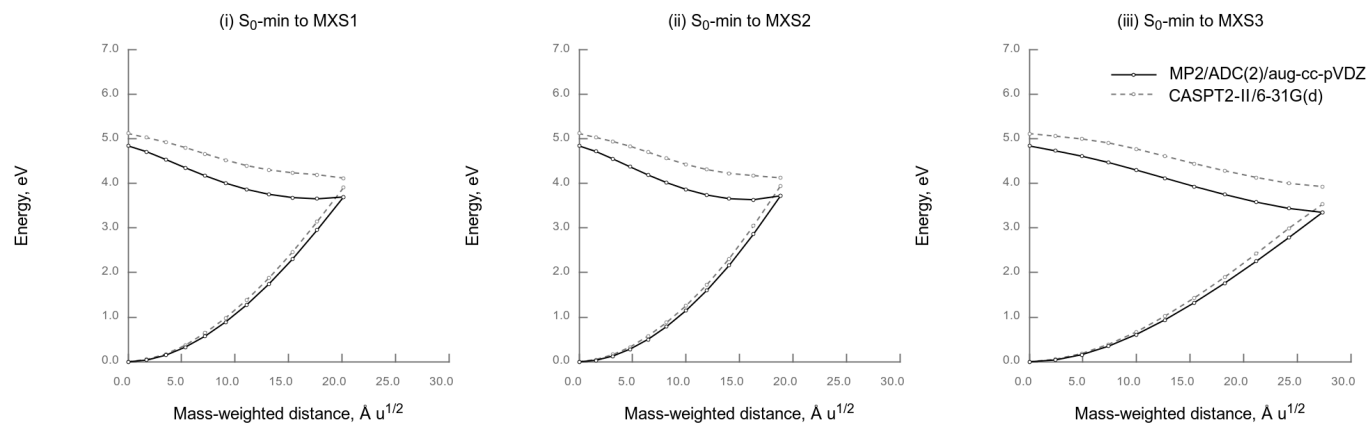


\subsection{Relaxation dynamics of photoexcited 9H-guanine}

The present simulations aimed to model the relaxation dynamics of $9 \mathrm{H}$-guanine in the gas phase following the irradiation of the low-energy region of the first photoabsorption band, where the $\mathrm{L}_{\mathrm{a}} \pi \pi^{*}$ state is the main absorbing state. Because the MP2/ADC $(2)$ cannot provide a formally correct description of crossings of excited states with the ground state, their scope was limited to the initial relaxation process from the Franck-Condon region to a CI seam with the ground state or, in a few trajectories, to the minimum on the $\mathrm{S}_{1}\left(\mathrm{n} \pi^{*}\right)$ state. The emphasis is therefore on the analysis of the molecular geometry on reaching the $\mathrm{S}_{1} / \mathrm{S}_{0}$ CI seam, and the excited-state lifetime. The dynamics was modeled using the trajectory surface hopping (TSH) variant ${ }^{24,25}$ of the nonadiabatic molecular dynamics method. The following two subsections describe the simulation setup.

\subsubsection{The trajectory surface hopping method}

Within the TSH method, the nuclear wavepacket of the system is represented by a so-called 'swarm,' or ensemble, of mutually independent semiclassical trajectories. In each trajectory, the nuclei are described using classical mechanics, while the electrons are treated quantum-mechanically. The time-dependent wavefunction $\Phi(\mathbf{r}, t)$ of the electrons is expressed as a linear combination of adiabatic electronic wavefunctions $\phi_{j}(\mathbf{r} ; \mathbf{R})$ :

$$
\Phi(\mathbf{r}, t)=\sum_{j} a_{j}(t) \phi_{j}(\mathbf{r} ; \mathbf{R}) \exp \left[-\frac{\mathrm{i}}{\hbar} \int E_{j}(\mathbf{R}) \mathrm{d} t\right]
$$

Here, $\quad \mathbf{R}=\mathbf{R}(t)$ denotes a classical trajectory followed by the nuclei. $\mathbf{r}$ is the position vector of the electrons, and the adiabatic electronic wavefunctions $\phi_{j}(\mathbf{r} ; \mathbf{R})$ are the solutions of the time-independent, 'clamped-nuclei' electronic Schroedinger equation, $\hat{H}_{\mathrm{el}}(\mathbf{r}, \mathbf{R}) \phi_{j}(\mathbf{r} ; \mathbf{R})=E_{j}(\mathbf{R}) \phi_{j}(\mathbf{r} ; \mathbf{R})$. The requirement that $\Phi(\mathbf{r}, t)$ satisfies the time-dependent electronic Schroedinger equation

$$
\hat{H}_{\mathrm{el}}(\mathbf{r}, \mathbf{R}) \Phi(\mathbf{r}, t)=\mathrm{i} \hbar \frac{\partial}{\partial t} \Phi(\mathbf{r}, t)
$$

leads to the following set of coupled differential equations for the time-evolution of the expansion coefficients $a_{j}(t)$ :

$$
\dot{a}_{i}=-\sum_{j} a_{j} C_{i j} \exp \left[-\frac{\mathrm{i}}{\hbar} \int\left(E_{j}-E_{i}\right) \mathrm{d} t\right]
$$

where $C_{i j}=\left\langle\phi_{i}\left|\frac{\partial}{\partial t}\right| \phi_{j}\right\rangle$ is the nonadiabatic coupling element between states $i$ and $j$. The square modulus $f_{j}(t)=\left|a_{j}(t)\right|^{2}$ of the expansion coefficient of state $j$ can be interpreted as the population of that state at time $t$.

During each simulated trajectory, at any time the system is considered to occupy some current adiabatic state $k$ from among the states which appear in the expansion (1). The nuclear dynamics are propagated according to Newton's equations of motion on the potential energy surface of that state:

$$
\ddot{\mathbf{R}}_{A}=-\frac{1}{M_{A}} \nabla_{A} E_{k}(\mathbf{R})
$$

Here, $\nabla_{A}$ denotes the gradient with respect to the Cartesian coordinates of nucleus $A$. The total energy of the system is taken as the sum of the energy $E_{k}$ of the current state and the kinetic energy $T$ of all atoms. Nonadiabatic effects are accounted for by allowing the system to undergo a switch (or 
'hop') between the current state and another adiabatic state, which then becomes the new current state. In the variant of the TSH method used in the present work, a hop is allowed to occur at each step of the nuclear dynamics, and the hopping probability is calculated by means of the fewest switches criterion of Tully, ${ }^{26}$ which aims to ensure that the fraction of the total number of trajectories evolving on any state $j$ is proportional to its population $f_{j}(t)=\left|a_{j}(t)\right|^{2}$ averaged over all trajectories, while minimizing the total number of hops per trajectory. Whenever a hop does occur, the nuclear momenta must be rescaled in such a way as to maintain the energy conservation of the system. In the implementation of the TSH method used in the present work, the nuclear momenta are rescaled uniformly according to

$$
\mathbf{p}^{(1)}=\mathbf{p}^{(0)} \sqrt{\frac{T^{(0)}+\left(E_{k}-E_{j}\right)}{T^{(0)}}}
$$

where the superscripts ${ }^{(0)}$ and ${ }^{(1)}$ denote quantities before and after the hop, respectively.

The TSH simulations were carried out using the implementation of the TSH method provided in the software package Newton-X version 1.4.0 ${ }^{27-29}$ which contains an interface to Turbomole. Adiabatic states from $\mathrm{S}_{1}$ to $\mathrm{S}_{5}$ were included in the expansion (1). Because the MP2/ADC(2) method does not provide a formally correct description of crossings of excited states with the ground state, the $\mathrm{S}_{0}$ state was not included in the expansion (1), and internal conversion to the ground state was not modeled explicitly. Instead, when the energy gap between the currently occupied state and the ground state in a given simulated trajectory decreased to below a threshold of $0.25 \mathrm{eV}$, the molecule was assumed to be undergoing internal conversion to the ground state, and at this point the simulated trajectory was terminated. The nuclear dynamics (equation 4) was propagated using the velocity Verlet integrator with a time step of $0.5 \mathrm{fs}$, while the time-evolution of the coefficients $a_{i}(t)$ (equation 3) was propagated with the default fifth-order integrator of Butcher ${ }^{30}$ and with a time step of $0.025 \mathrm{fs}$, using quantities interpolated between the classical time steps.

\subsubsection{Initial conditions}

The initial conditions for the TSH simulations were generated in such a way as to mimic photoexcitation in the low-energy region of the first absorption band. Firstly, the photoabsorption spectrum of $9 \mathrm{H}$-guanine was simulated using the semiclassical nuclear ensemble method. ${ }^{31}$ This method takes as input a set of $N_{\text {pts }}$ ground-state geometries of the molecule of interest $\left(\left\{\mathbf{R}_{i}\right\}\right)$, which are sampled either from a statistical distribution or from thermostatted molecular dynamics trajectories. Vertical excitation energies $\left(\Delta E_{0 n}\left(\mathbf{R}_{i}\right)\right)$ and associated oscillator strengths $\left(f_{0 n}\left(\mathbf{R}_{i}\right)\right)$ are computed at each of these geometries. Then, the molecular absorption cross-section $(\sigma(E))$ is calculated as:

$$
\sigma(E)=\frac{\pi e^{2} \hbar}{2 m c \varepsilon_{0} n_{r} E} \sum_{n=1}^{N_{\mathrm{fs}}} \frac{1}{N_{\mathrm{pts}}} \sum_{i=1}^{N_{\mathrm{pts}}} \Delta E_{0 n}\left(\mathbf{R}_{i}\right) f_{0 n}\left(\mathbf{R}_{i}\right) g_{\text {Gauss }}\left(E-\Delta E_{0 n}\left(\mathbf{R}_{i}\right), \delta\right)
$$

where $g_{\text {Gauss }}\left(E-\Delta E_{0 n}\left(\mathbf{R}_{i}\right), \delta\right)$ is a normalized Gaussian line-shape function whose purpose is to account for other sources of broadening, such as collisions and finite excited-state lifetime. $n_{r}$ denotes the refraction index of the medium, and $m$ is the electron rest mass. $N_{\mathrm{fs}}=8$ excited states were calculated at each of $N_{\mathrm{pts}}=500$ phase space points of $9 \mathrm{H}$-guanine, which had been sampled from the Wigner distribution of the $\mathrm{S}_{0}$-min structure in the vibrational ground state. Thus, the spectrum was built up from a total of $N_{\mathrm{fs}} \times N_{\mathrm{pts}}=4000$ spectral points. (In the present context, a phase space point is a set of nuclear positions and velocities. A spectral point can be thought of as a molecule at a given phase space point excited vertically to a specific excited state.) The broadening parameter in the line-shape functions were set to $\delta=0.1 \mathrm{eV}$. 
Figure S7: Photoabsorption spectrum of 9H-guanine in the gas phase, computed at the MP2/ADC(2)/cc-pVDZ level of theory. The black columns indicate the vertical excitation energies evaluated at the ground-state equilibrium geometry, and their relative heights are proportional to the corresponding oscillator strengths. The shaded area represents the energy interval from which initial conditions for the TSH simulations were sampled.

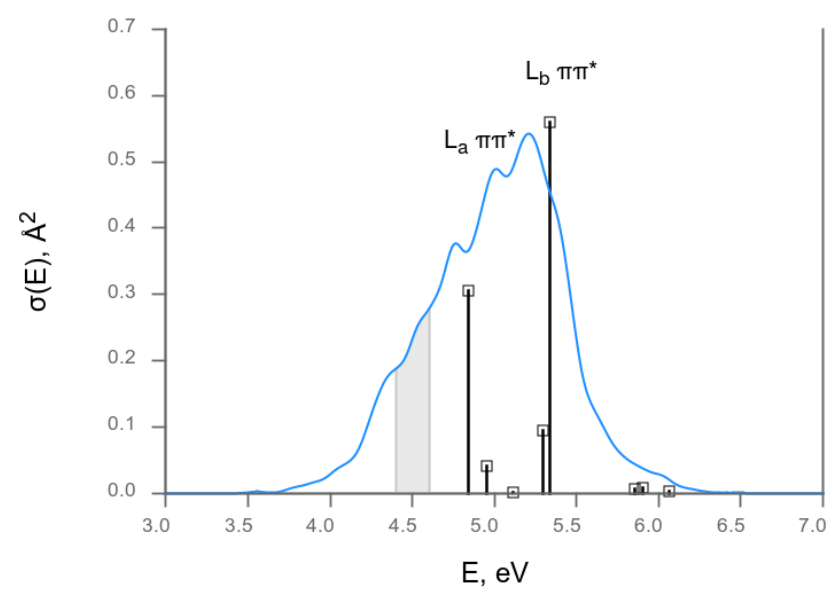

Afterwards, spectral points in the energy range of $4.5 \pm 0.1 \mathrm{eV}$ were sampled with probabilities proportional to their oscillator strengths, in order to serve as initial conditions for the TSH trajectories. A set of 36 spectral points were sampled in this manner.

The photoabsorption spectrum of $9 \mathrm{H}$-guanine calculated in the course of generating the initial conditions is shown in Figure S7. Absorption by the $\mathrm{L}_{\mathrm{a}} \pi \pi^{*}$ and $\mathrm{L}_{\mathrm{b}} \pi \pi^{*}$ states coalesces into a single absorption band. Because only eight excited states were included in the simulations, the simulated photoabsorption cross-section becomes vanishingly small at energies above around $6 \mathrm{eV}$, and no second absorption band appears.

\subsubsection{Results and discussion}

2 of the 36 simulated trajectories could not be completed because of convergence failures of the $\mathrm{ADC}(2)$ calculations. It was assumed that the occurrence of these convergence failures was not statistically correlated with the final outcome of the simulated trajectories, and therefore these 2 trajectories were simply discarded from further analysis. Figure S8 (a) depicts the fraction $f\left(\mathrm{~S}_{i}\right)$ of trajectories evolving in each adiabatic state during the remaining 34 simulated trajectories. At the time of the initial photoexcitation $(t=0)$, the majority of trajectories $(82 \%)$ were occupying the $\mathrm{S}_{1}$ state, with a minority $(18 \%)$ in the $\mathrm{S}_{2}$ state and none in the higher adiabatic states. During the first $50 \mathrm{fs}$ after the photoexcitation, the population of the $\mathrm{S}_{2}$ state was largely transferred to the $\mathrm{S}_{1}$ state, following which the $\mathrm{S}_{2}$ state was occupied only sporadically.

The earliest instance of internal conversion to the ground state occurred at $t=33.5 \mathrm{fs}$ in a trajectory in which the molecule hopped from the initially excited $\mathrm{L}_{\mathrm{a}} \pi \pi^{*}$ state to an excited state of $\pi \sigma^{*}$ character, and subsequently underwent hydrogen elimination from the $\mathrm{N} 9-\mathrm{H}$ bond. The onset of internal conversion near the $\mathrm{L}_{\mathrm{a}} \pi \pi^{*} / \mathrm{gs}$ CI seam took place roughly 80 fs after the initial photoexcitation, and by $t=700 \mathrm{fs}$, the remaining 33 of the simulated trajectories had undergone internal conversion in the vicinity of this CI seam. 
Figure S8: (a) Fraction of simulated trajectories occupying each adiabatic state. Here, $f\left(\mathrm{~S}_{0}\right)$ represents trajectories in which the energy gap between the occupied state and the $\mathrm{S}_{0}$ state decreased to below $0.25 \mathrm{eV}$, and which were therefore considered to have undergone internal conversion to the ground state.

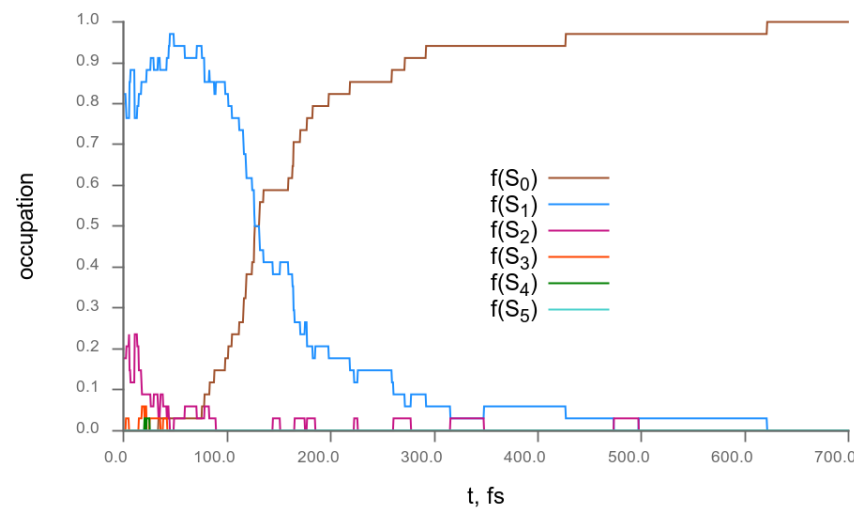

(b) Total excited-state population, $\sum_{j=1}^{5} f\left(\mathrm{~S}_{j}\right)$, and delayed exponential fit.

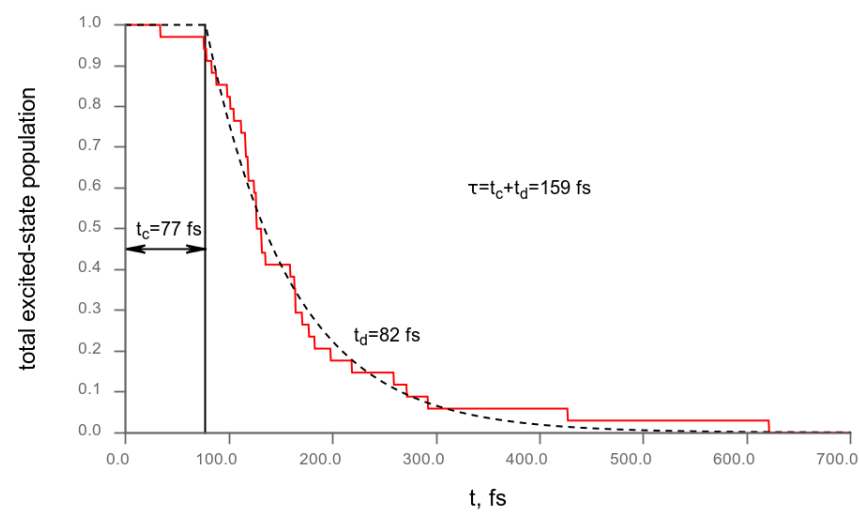

In some simulated trajectories, the molecule temporarily entered the $\mathrm{n}_{\mathrm{O}} \pi^{*}$ region of the $\mathrm{S}_{1}$ adiabatic state, which was verified through the inspection of the EDDM of the currently occupied addiabatic state, and by monitoring the length of the C6-O bond (when the molecule is in the $\mathrm{n}_{\mathrm{O}} \pi^{*}$ state, this bond rapidly elongates). However, in no simulated trajectory did the molecule remain trapped in the minimum on the $\mathrm{S}_{1}\left(\mathrm{n}_{\mathrm{O}} \pi^{*}\right)$ state $\left(\mathrm{S}_{1}-\mathrm{n}_{\mathrm{O}} \pi^{*}\right.$-min) for more than a few hundred femtoseconds. Thus, hydrogen elimination and trapping in the $\mathrm{S}_{1}\left(\mathrm{n}_{\mathrm{O}} \pi^{*}\right)$ state play only minor roles in the relaxation dynamics of $9 \mathrm{H}$-guanine, and the vast majority the excited-state population can be described as decaying with a single time constant through the direct diabatic pathway on the $\mathrm{L}_{\mathrm{a}} \pi \pi^{*}$ state.

Following the example of Barbatti and coworkers, ${ }^{15}$ we have least-square fitted a delayed exponential decay function $p(t)$ to the total excited-state population during the simulated trajectories:

$$
p(t)= \begin{cases}1 & \text { for } t \leq t_{\mathrm{c}} \\ \exp \left[-\left(t-t_{\mathrm{c}}\right) / t_{\mathrm{d}}\right] & \text { for } t>t_{\mathrm{c}}\end{cases}
$$

Here, $t_{\mathrm{d}}$ is the exponential decay time and $t_{\mathrm{c}}$ is the delay between the initial photoexcitation and the onset of exponential decay. Both these variables are parameter of the fit. The excited-state lifetime 
is estimated as $\tau=t_{\mathrm{d}}+t_{\mathrm{c}}$. We have obtained $t_{\mathrm{c}}=77 \mathrm{fs}, t_{\mathrm{d}}=82 \mathrm{fs}$ and $\tau=159 \mathrm{fs}$, which is somewhat shorter but still in reasonably good agreement with the value of $\tau=224$ fs reported by Barbatti et al.

Figure 9 (a) is a scatter plot of the Hill-Reilly puckering coordinates of the six-membered ring at the time of internal conversion. Except for the single trajectory in which the molecule decayed to the ground state through hydrogen elimination, the data points are mostly clustered in two regions of the plot: in between the points corresponding to the ideal ${ }^{2} \mathrm{E}$ and ${ }^{2,5} \mathrm{~B}$ conformations, and in between the points representing the ideal $\mathrm{E}_{2}$ and $\mathrm{B}_{2,5}$ conformations. As illustrated in Figure 9 (b), the separation of data points into two distinct clusters occurs because in some trajectories, atoms $\mathrm{C} 2$ and $\mathrm{C} 5$ are displaced above the plane of the ring, while in others, they are displaced below the plane of the ring, and the Hill-Reilly scheme distinguishes between these two situations. This aside, most of the trajectories that deactivate near the $\mathrm{L}_{\mathrm{a}} \pi \pi^{*} / \mathrm{gs}$ CI seam do so in a compact region of the conformation space, between ideal boat-type and envelope- type conformations.

Despite the fact that MXS3 is the global minimum along the $\mathrm{L}_{\mathrm{a}} \pi \pi^{*} / \mathrm{gs}$ CI seam at the MP2/ADC(2)/aug-cc-pVDZ level, few trajectories encountered the $\mathrm{L}_{\mathrm{a}} \pi \pi^{*} /$ gs CI seam at MXS3-like geometries featuring a significant displacement of the amino group from the plane of the six-membered ring. This is in contrast to the previously reported simulations, in which such geometries were common (reported to occur in $40 \%$ of trajectories at the OM2/MRCI level ${ }^{13}$ and $28 \%$ of trajectories at the MRCIS level ${ }^{15}$ ). Since MXS3 does exist at the MP2/ADC(2) level, this observation does not indicate a qualitative failure of the MP2/ADC(2) method, and is likely a only manifestation of minor deviations between PESs predicted by MP2/ADC(2) and the other two electronic structure methods.

In order to accompany this narrative, a movie showing a representative simulated trajectory (jp6b04723_si_001.avi) is provided as part of the Supporting Information. The animation includes the EDDM of the currently occupied state, plotted in the form of isosurfaces with isovalues of $\pm 0.005 e / a_{0}{ }^{3}$, throughout the entire trajectory. At the bottom of the animation is a graph showing the energies of the adiabatic states included in the expansion (1). The currently occupied state is indicated by a black bead. The molecule follows the direct diabatic pathway on the $\mathrm{L}_{\mathrm{a}} \pi \pi^{*}$ state from the Franck-Condon region to the $\mathrm{L}_{\mathrm{a}} \pi \pi^{*} / \mathrm{gs}$ CI seam. At $t=163.5 \mathrm{fs}$, the molecule undergoes internal conversion to the ground state (in the sense that at this time, the energy gap between the $\mathrm{S}_{1}$ and $\mathrm{S}_{0}$ states decreases to below $0.25 \mathrm{eV}$ ), and the simulated trajectory ends.

To conclude, our simulations have shown that the photorelaxation mechanism predicted by $\mathrm{MP} 2 / \mathrm{ADC}(2)$ is in qualitative agreement with previous simulation studies. ${ }^{13,15}$ This observation paved the way for the application of this computationally efficient electronic structure method to larger systems comprising the quanine moiety, and in particular the fluorescent guanine analogue $8 \mathrm{vG}$. 
Figure S9: (a) Scatter plot of Hill-Reilly puckering coordinates at the time of internal conversion to the ground state. The vertical black lines are a guide to the eye, and connect the data points with the $\theta_{2}=-90^{\circ}$ plane. The positions of canonical $\mathrm{E}_{2},{ }^{2} \mathrm{E}, \mathrm{B}_{2,5}$, and ${ }^{2,5} \mathrm{~B}$ geometries are marked with red arrows. The single trajectory in which the molecule decayed through hydrogen elimination is marked with an asterisk.

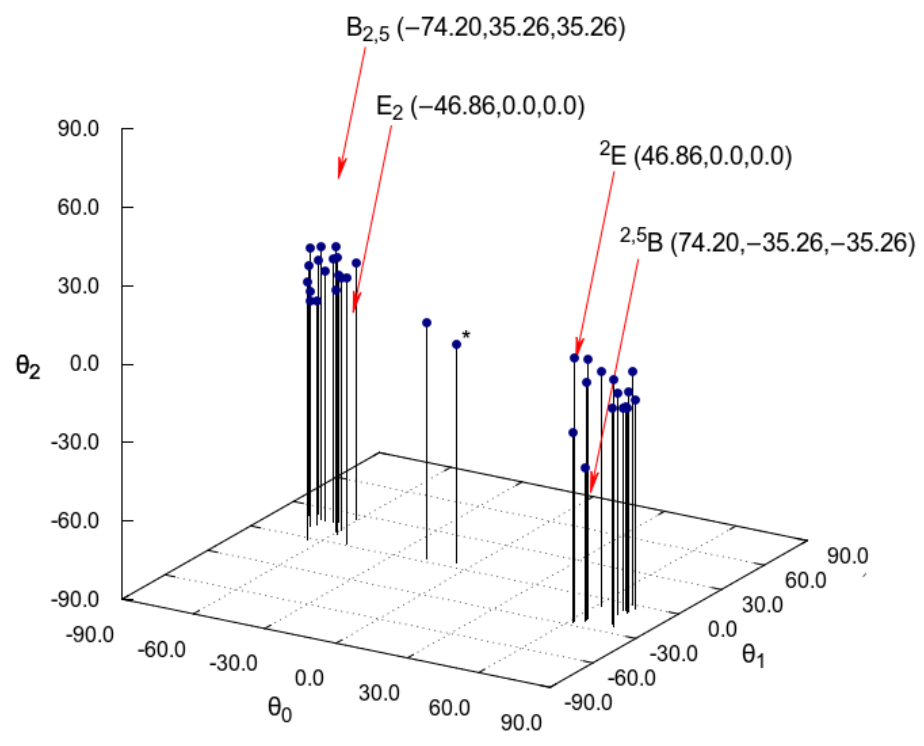

(b) Two representative geometries of $9 \mathrm{H}$-guanine at the time of internal conversion to the electronic ground state. In the structure on the left, atoms $\mathrm{C} 2$ and $\mathrm{C} 5$ are displaced above the plane of the six-membered ring. It is characterized by $\left(\theta_{0}, \theta_{1}, \theta_{2}\right)=\left(64.70^{\circ},-23.78^{\circ},-31.43^{\circ}\right)$, and so it is close to the ideal ${ }^{2,5} \mathrm{~B}$-type conformation. In turn, in the structure on the right, atoms $\mathrm{C} 2$ and $\mathrm{C} 5$ are displaced below the plane of the ring. The Hill-Reilly puckering coordinates adopt values of $\left(\theta_{0}, \theta_{1}, \theta_{2}\right)=\left(-58.83^{\circ}, 8.76^{\circ}, 16.12^{\circ}\right)$, such that it lies between the ideal $\mathrm{E}_{2}$ and $\mathrm{B}_{2,5}$ conformations.
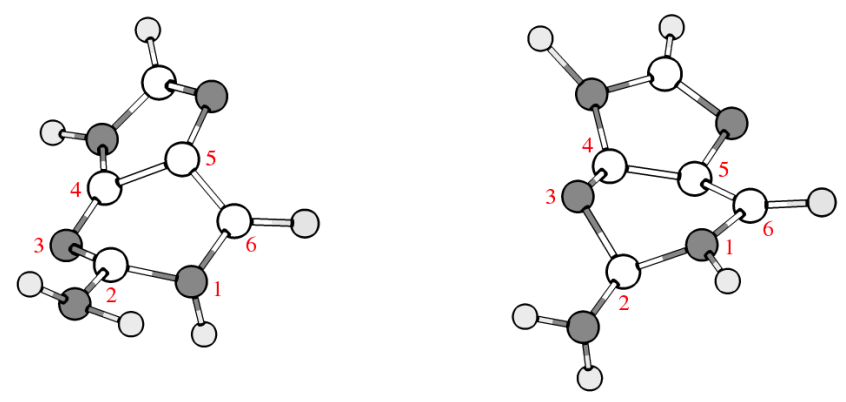


\section{Conformational and tautomeric preference of $8 \mathrm{vG}$}

The present section discusses conformational isomerism and tautomerism of $8 \mathrm{vG}$. In experimental studies, $8 \mathrm{vG}$ has been used in nucleoside form or as a building block of peptide nucleic acid (PNA), in either case substituted with an $\mathrm{sp}^{3}$-hybridized carbon atom at position $\mathrm{N} 9$, and in aqueous solution. Accordingly, in order to mimic experimental conditions, the conformational and tautomeric equilibria were simulated for the model molecule 8-vinyl-9-methylguanine (8v9MeG).

As illustrated in Figure $\mathrm{S} 10,8 \mathrm{v} 9 \mathrm{MeG}$ exhibits syn-anti conformational isomerism with respect to rotation around the $\mathrm{C} 9-\mathrm{C} 11$ bond, and may also exist in several tautomeric forms.

Equilibrium geometries of all isomers depicted in Figure S10 were optimized and confirmed to correspond to potential energy minima through the calculation of the normal modes of the molecule. The calculations were performed at the density functional theory (DFT) level of theory within Gaussian 09 Revision A.02. ${ }^{32}$ The B3LYP exchange-correlation functional ${ }^{33,34}$ was applied in combination with the high-quality def2-TZVPP basis set. ${ }^{35}$ The interaction of the molecule with bulk solvent (water, dielectric constant $\epsilon=78.3553$ ) was modeled using the continuous surface charge implementation of the polarizable continuum model (CSC-PCM). ${ }^{36,37}$

The relative energies of the various isomeric forms of $8 \mathrm{v} 9 \mathrm{MeG}$, and estimated populations at room temperature, are listed in Table $\mathrm{S} 4$, from where it can be seen that the dominant isomer under normal conditions is the syn keto- $1 \mathrm{H}$ form, which makes up around $91 \%$ of the total population. The second most prevalent isomer is the anti keto- $1 \mathrm{H}$, with a population of around $9 \%$. The populations of the remaining tautomers are negligibly small (less than 1\%). Because of the predominance of the isomer of $8 \mathrm{v} 9 \mathrm{MeG}$, in our study of the photochemistry of $8 \mathrm{vG}$ we took into consideration only its syn keto-1H tautomer.

Table S4: Energies $(E)$, Gibbs free energies $(G)$ of isomers of $8 \mathrm{v} 9 \mathrm{MeG}$ at a temperature of $298 \mathrm{~K}$ and pressure of $1 \mathrm{~atm}$., and their estimated populations. The energy and Gibbs free energy values are quoted relative to the lowest energy syn keto- $1 \mathrm{H}$ isomer. The energy values include zero-point vibrational corrections.

\begin{tabular}{|l|l|l|l|}
\hline Tautomer & $E, \mathrm{eV}$ & $G, \mathrm{eV}$ & population \\
\hline syn keto-1H & 0 & 0 & 0.91 \\
syn keto-3H & 0.445 & 0.462 & $<0.01$ \\
syn enol I & 0.299 & 0.300 & $<0.01$ \\
syn enol II & 0.333 & 0.334 & $<0.01$ \\
anti keto-1H & 0.048 & 0.059 & 0.09 \\
anti keto-3H & 0.511 & 0.518 & $<0.01$ \\
anti enol I & 0.350 & 0.361 & $<0.01$ \\
anti enol II & 0.385 & 0.396 & $<0.01$ \\
\hline
\end{tabular}


Figure S10: Low-energy conformational and tautomeric isomers of $8 \mathrm{v} 9 \mathrm{MeG}$.

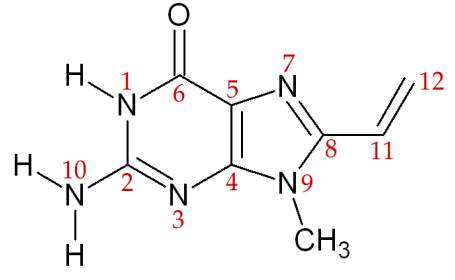

(a) syn keto- $1 \mathrm{H}$<smiles>C=Cc1nc2c(O)nc(N)nc2n1C</smiles>

(c) syn enol I<smiles>C=Cc1nc2c(=O)[nH]c(N)nc2n1C</smiles>

(e) anti keto- $1 \mathrm{H}$<smiles>C=Cc1nc2c(O)nc(N)nc2n1C</smiles>

(g) anti enol I<smiles>C=Cc1nc2c(=O)nc(N)[nH]c2n1C</smiles>

(b) syn keto-3H<smiles>C=Cc1nc2c(O)nc(N)nc2n1C</smiles>

(d) syn enol II<smiles>C=Cc1nc2c(=O)nc(N)[nH]c2n1C</smiles>

(f) anti keto-3H<smiles>C=Cc1nc2c(O)nc(N)nc2n1C</smiles>

(h) anti enol II 


\section{Influence of solvating water molecules on the excited electronic states of $8 \mathrm{vG}$}

In addition to the calculations making use of the COSMO implicit solvation model reported in the main body of the present work, we have also explored the effect of hydrogen bonding with solvating water molecules on the ground- and excited-state structures of $8 \mathrm{vG}$. This section gives an outline of the results of these simulations.

\subsection{Simulation setup}

As in the preceding section, simulations were carried out with $8 \mathrm{v} 9 \mathrm{MeG}$ as a model of the deoxyribonucleoside form of $8 \mathrm{vG}$. The solvation shell around the $8 \mathrm{v} 9 \mathrm{MeG}$ molecule was constructed in such a way as to saturate most of its hydrogen bonding sites with as few water molecules as possible, leading to the complex of $8 \mathrm{v} 9 \mathrm{MeG}$ with five water molecules $(8 \mathrm{v} 9 \mathrm{MeG}-5 \mathrm{~W})$ whose structure is shown schematically in Figure S11. It features three water molecules hydrogen-bonded to the Watson-Crick face, one to the sugar edge, and one to the Hoogsteen face.

Figure S11: The 8v9MeG-5W complex, which was used to explore the interaction of the $8 \mathrm{vG}$ chromophore with solvating water molecules.

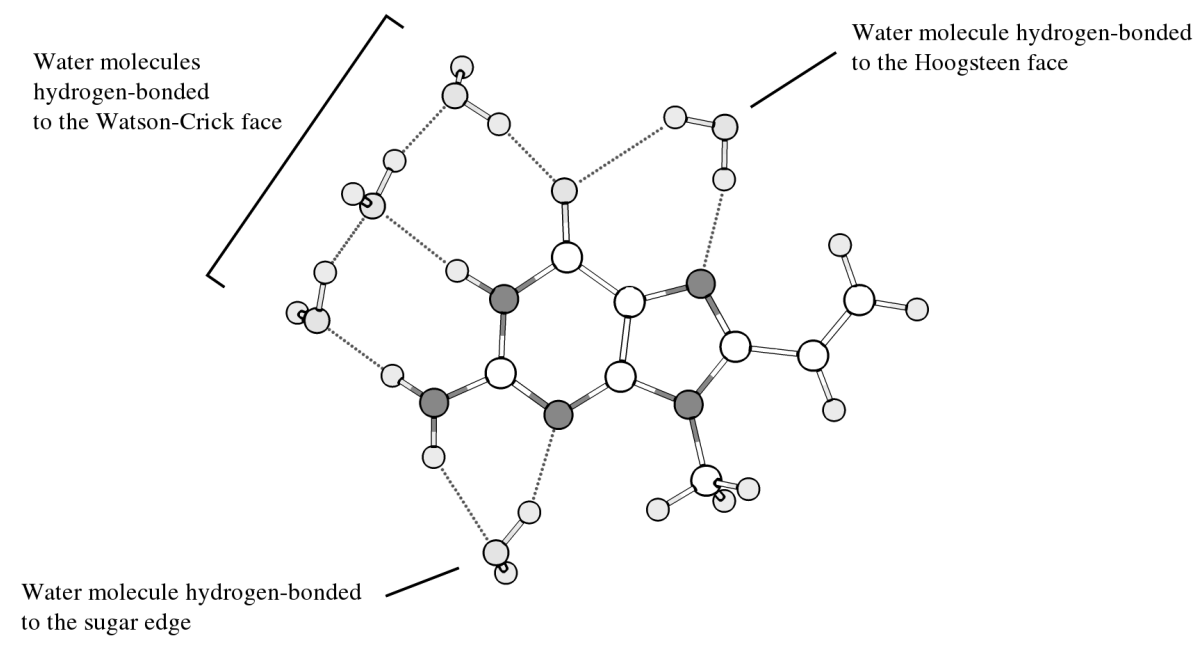

The computational parameters used in the simulations of the $8 \mathrm{v} 9 \mathrm{MeG}-5 \mathrm{~W}$ cluster were generally the same as for the $8 \mathrm{vG}$ molecule in the gas phase (see Section 2.1 in the main body of the present work), except that the description of hydrogen bonding with the solvating water molecules necessitated the use of a basis set augmented with diffuse functions. As a compromise between accuracy and computational efficiency, we applied the jun-cc-pVDZ basis set, which is one of the "calendar basis sets" proposed by Papajak et al. ${ }^{38}$ These basis sets are derived through the systematic elimination of diffuse basis functions from the aug-cc-pVnZ basis sets. In particular, the jun-cc-pVDZ basis set is obtained from aug-cc-pVDZ by deleting the diffuse d subshell from the second-row elements, as well as all diffuse functions from hydrogen. Thus, only $\mathrm{s}$ and $\mathrm{p}$ diffuse functions on the second-row elements are retained. Papajak et al. ${ }^{38}$ have shown that this augmentation scheme performs very closely to the original aug-cc-pVDZ basis set for hydrogen bond lengths and energies.

The corresponding auxiliary basis set for jun-cc-pVDZ was generated from the default auxiliary basis 
set for aug-cc-pVDZ by removing all diffuse functions from hydrogen, and diffuse f-type functions from the second-row elements.

All geometry optimizations were carried out in Cartesian coordinates.

\subsection{Results and discussion}

Figure S12 shows the equilibrium geometries of the isolated $8 \mathrm{v} 9 \mathrm{MeG}$ molecule as optimized with the use of the jun-cc-pVDZ basis set, while the relative energies of these structures are listed in Table S5. Aside from the fact that equilibrium bond lengths obtained with the jun-cc-pVDZ basis set are systematically longer than with cc-pVTZ, these geometries are largely similar to those obtained for $8 \mathrm{vG}$ with the cc-pVTZ basis set (see Figures 3 and 5 in the main body of the present work). The only point of note is that in the $\mathrm{S}_{1}$-ICT-min equilibrium geometry of $8 \mathrm{v} 9 \mathrm{MeG}$ obtained with the jun-cc-pVDZ basis set, the five-membered ring is slightly puckered at atom C8, causing the vinyl group to point away from the plane of the purine moiety. We have no explanation for this result, which may be a basis set incompleteness-related artifact due to the relatively small size of the jun-cc-pVDZ basis set. We have excluded the possibility that it is the methyl group at nitrogen N9 which is responsible for this puckering, because it is also seen when the $\mathrm{S}_{1}$-ICT-min structure of $8 \mathrm{vG}$ is reoptimized at the MP2/ADC(2)/jun-cc-pVDZ level.

Table S5: Characterization of equilibrium geometries of $8 \mathrm{v} 9 \mathrm{MeG}$ and the $8 \mathrm{v} 9 \mathrm{MeG}-5 \mathrm{~W}$ complex as optimized at the MP2/ADC(2)/jun-cc-pVDZ level of theory. $E$ is the energy of a given electronic state relative to the ground state at the lowest-energy ground-state equilibrium geometry, while $f$ denotes the oscillator strength of the $\mathrm{S}_{0} \rightarrow \mathrm{S}_{n}$ transition. No zero-point vibrational energy corrections were applied.

\begin{tabular}{|c|c|c|c|c|}
\hline System & Structure & Electronic state & $E, \mathrm{eV}$ & $f$ \\
\hline $8 \mathrm{v} 9 \mathrm{MeG}$ & $\begin{array}{l}\mathrm{S}_{0}-\min \\
\mathrm{S}_{1}-\mathrm{ICT}-\min \\
\mathrm{S}_{1}-\mathrm{n}_{\mathrm{O}} \pi^{*} \text {-min }\end{array}$ & $\begin{array}{l}\mathrm{S}_{0} \\
\mathrm{~S}_{1}\left(\mathrm{ICT} \pi \pi^{*}\right) \\
\mathrm{S}_{2}\left(\mathrm{~L}_{\mathrm{a}} \pi \pi^{*}\right) \\
\mathrm{S}_{0} \\
\mathrm{~S}_{1}\left(\mathrm{ICT} \pi \pi^{*}\right) \\
\mathrm{S}_{2}\left(\mathrm{~L}_{\mathrm{a}} \pi \pi^{*}\right) \\
\mathrm{S}_{0} \\
\mathrm{~S}_{1}\left(\mathrm{n}_{\mathrm{O}} \pi^{*}\right) \\
\mathrm{S}_{2}\left(\mathrm{~L}_{\mathrm{a}} \pi \pi^{*}\right)\end{array}$ & $\begin{array}{l}0 \\
4.396 \\
4.736 \\
0.429 \\
4.032 \\
4.717 \\
1.788 \\
3.938 \\
5.282 \\
\end{array}$ & $\begin{array}{l}0.583 \\
0.064 \\
\\
0.576 \\
0.011 \\
\\
4 \times 10^{-4} \\
0.113\end{array}$ \\
\hline $8 \mathrm{v} 9 \mathrm{M}$ & $\begin{array}{l}\mathrm{S}_{0}-\min 1 \\
\mathrm{~S}_{0}-\min 2 \\
\mathrm{~S}_{1}-\mathrm{ICT}-\min \end{array}$ & $\begin{array}{l}\mathrm{S}_{0} \\
\mathrm{~S}_{1}\left(\operatorname{ICT} \pi \pi^{*}\right) \\
\mathrm{S}_{2}\left(\mathrm{~L}_{\mathrm{a}} \pi \pi^{*}\right) \\
\mathrm{S}_{0} \\
\mathrm{~S}_{1}\left(\mathrm{ICT} \pi \pi^{*}\right) \\
\mathrm{S}_{2}\left(\mathrm{~L}_{\mathrm{a}} \pi \pi^{*}\right) \\
\mathrm{S}_{0} \\
\mathrm{~S}_{1}\left(\mathrm{ICT} \pi \pi^{*}\right) \\
\mathrm{S}_{2}\left(\mathrm{~L}_{\mathrm{a}} \pi \pi^{*}\right)\end{array}$ & $\begin{array}{l}0.002 \\
4.302 \\
4.720 \\
0 \\
4.311 \\
4.719 \\
0.399 \\
3.881 \\
4.749 \\
\end{array}$ & $\begin{array}{l}0.487 \\
0.217 \\
\\
\\
0.497 \\
0.212 \\
\\
\\
0.473 \\
0.197\end{array}$ \\
\hline
\end{tabular}


Figure S12: Equilibrium geometries of the $8 \mathrm{v} 9 \mathrm{MeG}$ molecule as obtained at the MP2/ADC(2)/jun-cc-pVDZ level of theory. Below each structure is a representation of its electric dipole moment vector. Selected bond lengths are given in units of $\AA$. The $\mathrm{S}_{1}$-ICT-min structure features a puckered atom $\mathrm{C} 8$, which is possibly an artifact due to basis set incompleteness, and consequently the heavy-atom skeleton appears bent.
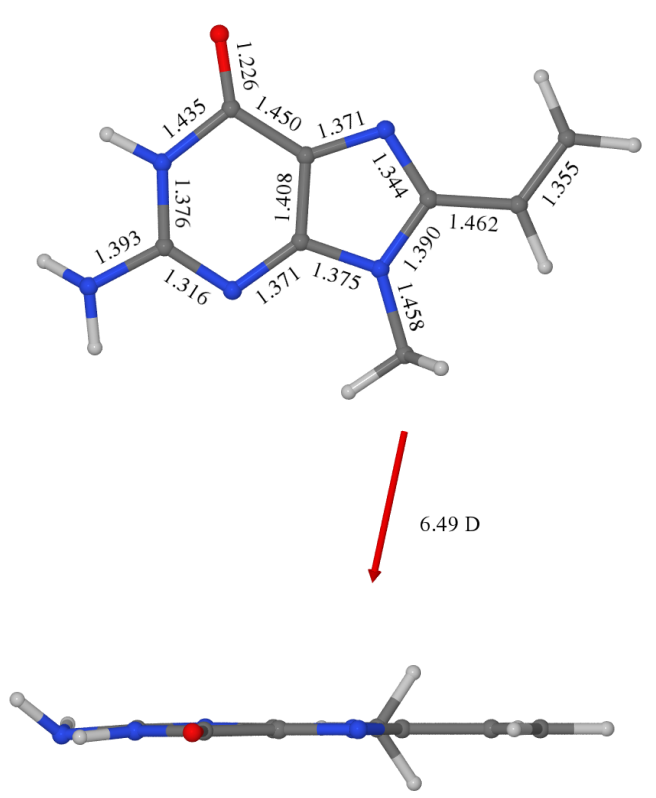

(a) $\mathrm{S}_{0}-\mathrm{min}$
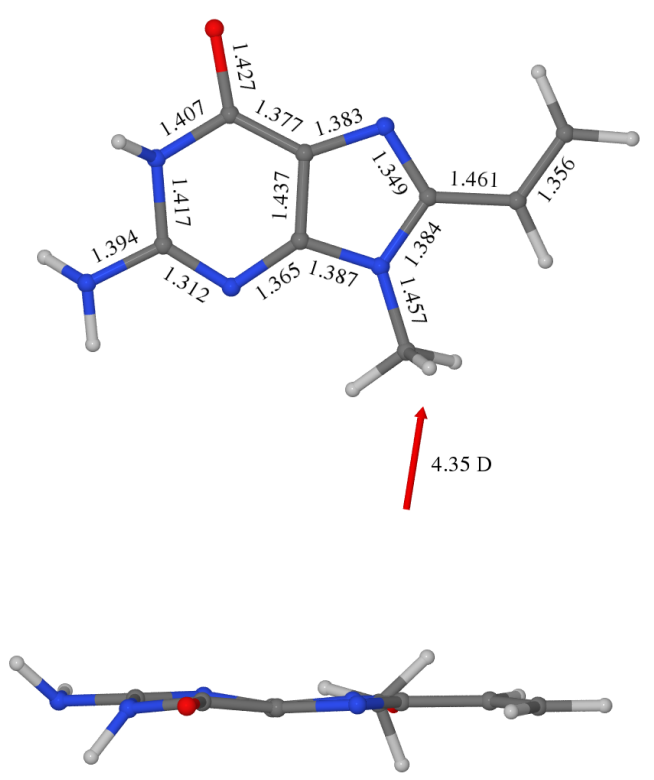

(c) $\mathrm{S}_{1}-\mathrm{n}_{\mathrm{O}} \pi^{*}$-min
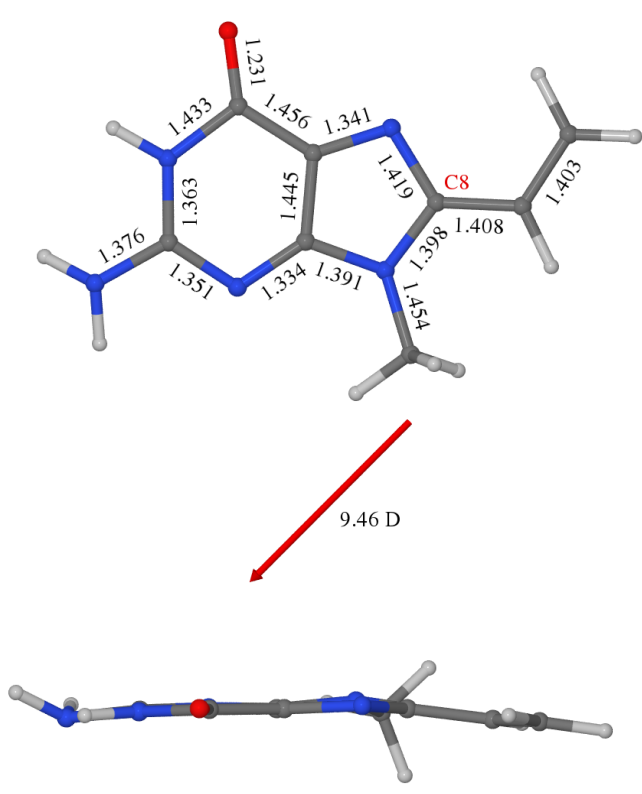

(b) $\mathrm{S}_{1}$-ICT-min 
Two minima with partially different hydrogen bonding patterns were found on the ground-state potential energy surface of the 8v9MeG-5W complex, and their structures are shown in Figure S13. In the minimum labeled $\mathrm{S}_{0}$-min1, the hydrogen bond between the carbonyl oxygen of $8 \mathrm{v} 9 \mathrm{MeG}$ and the water molecule at the Hoogsteen face is unusually long $(2.485 \AA)$, which suggests that this bond is very weak. We therefore sought and optimized a second equilibrium geometry $\left(\mathrm{S}_{0}-\min 2\right)$ in which this bond is broken completely, allowing that water molecule to form a near-linear hydrogen bond with nitrogen N7. The two minima turn out to be very close in energy, with $\mathrm{S}_{0}$-min2 being lower than $\mathrm{S}_{0}$-min1 by only $0.002 \mathrm{eV}$.

We have located and optimized a single minimum on the $S_{1}$ potential energy surface of the 8v9MeG-5W complex, where the $\mathrm{S}_{1}$ state is ICT $\pi \pi^{*}$ in character. We label it the $\mathrm{S}_{1}$-ICT-min structure of the 8v9MeG-5W complex, and present its geometry in Figure S14, along with the EDDMs of the $\mathrm{S}_{1}$ and $\mathrm{S}_{2}$ states. The existence of a minimum on the $\mathrm{S}_{1}$ (ICT $\pi \pi^{*}$ ) state of the $8 \mathrm{v} 9 \mathrm{MeG}-5 \mathrm{~W}$ complex, as well as in the isolated $8 \mathrm{vG}$ molecule, provides additional support for our finding that it is the ICT $\pi \pi^{*}$ state that is responsible for trapping the excited-state population, and fluorescence. The arrangement of the water molecules hydrogen-bonded to the Watson-Crick face and the sugar edge of the nucleobase is similar as in the ground-state equilibrium geometries $\mathrm{S}_{0}$-min1 and $\mathrm{S}_{0}$-min2, barring relatively minor changes in hydrogen bond lengths. This is unsurprising, since the ICT $\pi \pi^{*}$ state is mostly localized on the five-membered ring and the vinyl group, and to a smaller extent on the atoms making up the Watson-Crick face and nitrogen N3 which is located along the sugar edge.

In contrast, hydrogen bonding to the water molecule at the Hoogsteen face is clearly different in the $\mathrm{S}_{1}$-ICT-min structure of the $8 \mathrm{v} 9 \mathrm{MeG}$ complex than in either of its ground-state equilibrium geometries. Whereas in both $\mathrm{S}_{0}-\min 1$ and $\mathrm{S}_{0}$-min2, this water molecule is positioned roughly in the plane of $8 \mathrm{v} 9 \mathrm{MeG}$ molecule, in $\mathrm{S}_{1}$-ICT-min it is strongly displaced from the plane of the 8v9MeG molecule while remaining hydrogen-bonded to the carbonyl oxygen and nitrogen N7. This displacement is presumably caused by the fact that the $\mathrm{S}_{1}$ (ICT $\left.\pi \pi^{*}\right)$ state is significantly localized on the carbonyl oxygen and nitrogen $\mathrm{N} 7$ of the $8 \mathrm{vG}$ chromophore, changing their hydrogen-bonding preferences. In particular, the out-of-plane p orbital of nitrogen N7 accepts electron density in the $\mathrm{S}_{1}\left(\mathrm{ICT} \pi \pi^{*}\right)$ state, and it may therefore become more energetically favorable for it to form out-of-plane hydrogen bonds.

Since the carbon atoms of the vinyl group accept a substantial amount of electron density in the $\mathrm{S}_{1}$ (ICT $\pi \pi^{*}$ ) state, we also hypothesized that one or both might act as hydrogen bond acceptors. However, this was not borne out by calculations: attempts to optimize a minimum on the $\mathrm{S}_{1}$ state with a water molecule hydrogen-bonded to either of the vinyl group carbons resulted in the water molecule migrating along the $\pi$-bonding system of the $8 \mathrm{v} 9 \mathrm{MeG}$ molecule to hydrogen-bond with nitrogen N7 instead. We may conclude that the vinyl group is not a good hydrogen bond acceptor in the $\mathrm{S}_{1}\left(\mathrm{ICT} \pi \pi^{*}\right)$ state.

Finally, it is noteworthy that at the $\mathrm{S}_{1}$-ICT-min structure of the $8 \mathrm{v} 9 \mathrm{MeG}-5 \mathrm{~W}$ complex, the energy separation between the $\mathrm{S}_{1}\left(\mathrm{ICT} \pi \pi^{*}\right)$ and $\mathrm{S}_{2}\left(\mathrm{~L}_{\mathrm{a}} \pi \pi^{*}\right)$ states, at $0.868 \mathrm{eV}$, is only slightly larger than at the $\mathrm{S}_{1}$-ICT-min structure of the isolated $8 \mathrm{v} 9 \mathrm{MeG}$ molecule $(0.685 \mathrm{eV})$. In contrast, as seen in Table 2 in the main body of the present work, the inclusion of solvent effects via the COSMO model increases the energy gap between these states at $\mathrm{S}_{1}$-ICT-min from $0.612 \mathrm{eV}$ to $1.231 \mathrm{eV}$. This observation suggests that the addition of a few explicit water molecules hydrogen-bonded to the $8 \mathrm{vG}$ fluorophore accounts for only a small part of the electrostatic stabilization of the $\mathrm{S}_{1}$ (ICT $\pi \pi^{*}$ ) state by the bulk aqueous solvent. Therefore, the application of a continuum solvent model, or possibly the combination of a continuum solvent model and explicit water molecules, seems necessary in order to recover the full effect of aqueous solvation on the relative stability of the ICT $\pi \pi^{*}$ and $\mathrm{L}_{\mathrm{a}} \pi \pi^{*}$ states. 
Figure S13: Equilibrium ground-state geometries of the $8 \mathrm{v} 9 \mathrm{MeG}-5 \mathrm{~W}$ complex. $\mathrm{S}_{0}-\min 2$ is marginally (by $0.002 \mathrm{eV}$ ) lower in energy than $\mathrm{S}_{0}$-min1. Hydrogen bond lengths are given in units of $\AA$.
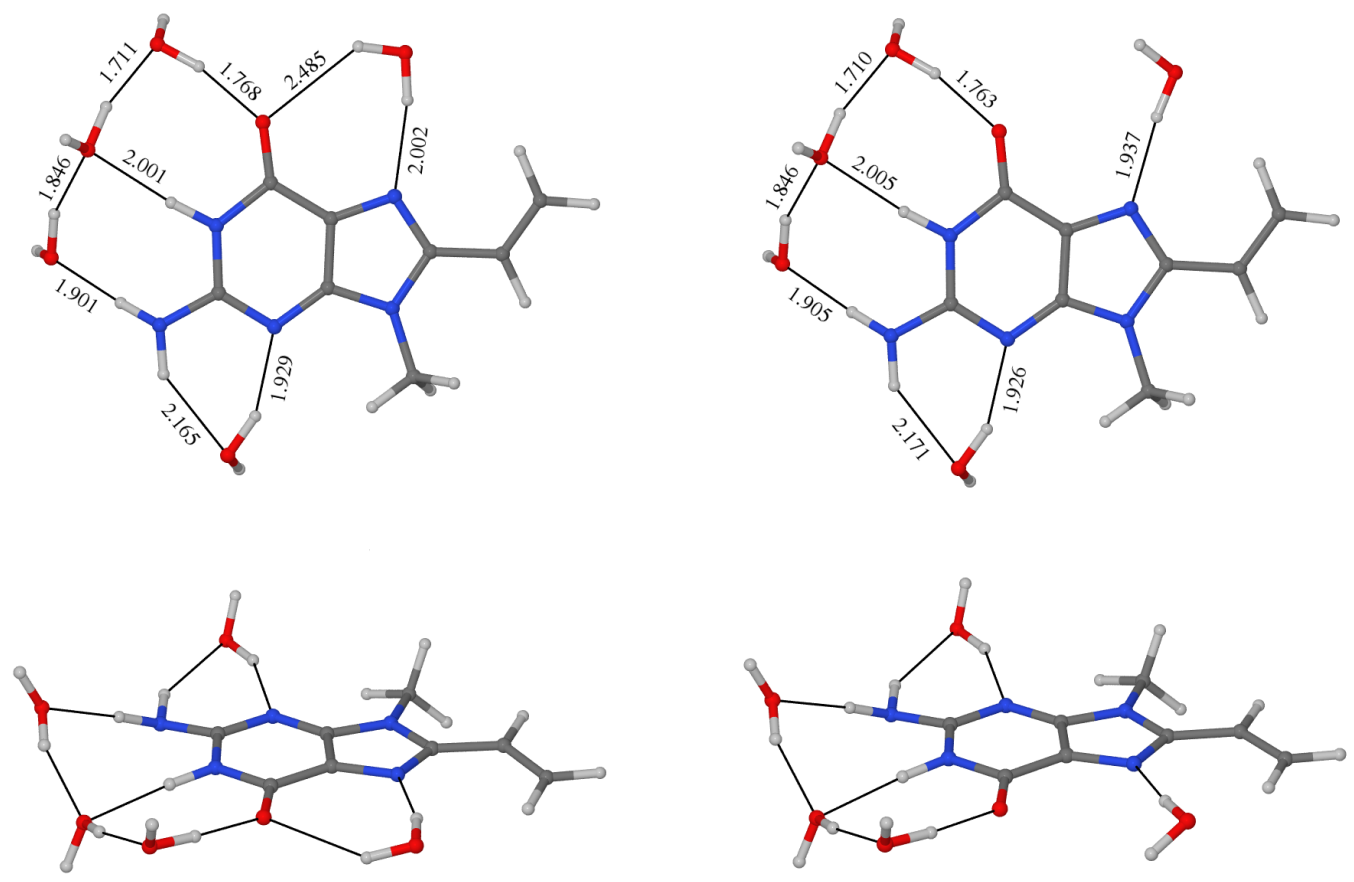

(a) $8 \mathrm{vG}-5 \mathrm{~W} \mathrm{~S}_{0}-\min 1$

(b) $8 \mathrm{vG}-5 \mathrm{~W} \mathrm{~S}_{0}-\mathrm{min} 2$ 
Figure S14: (a) The $\mathrm{S}_{1}$-ICT-min geometry of the $8 \mathrm{v} 9 \mathrm{MeG}-5 \mathrm{~W}$ complex. Hydrogen bond lengths are given in units of $\AA$. Unlike in either of the two ground-state equilibrium geometries, the water molecule hydrogen-bonded to the oxygen atom and nitrogen N7 is substantially displaced from the plane of the $8 \mathrm{v} 9 \mathrm{MeG}$ molecule. We attribute tentatively attribute this observation to the donation of electron density into the out-of-plane p orbital of nitrogen N7 in the ICT $\pi \pi^{*}$ state.
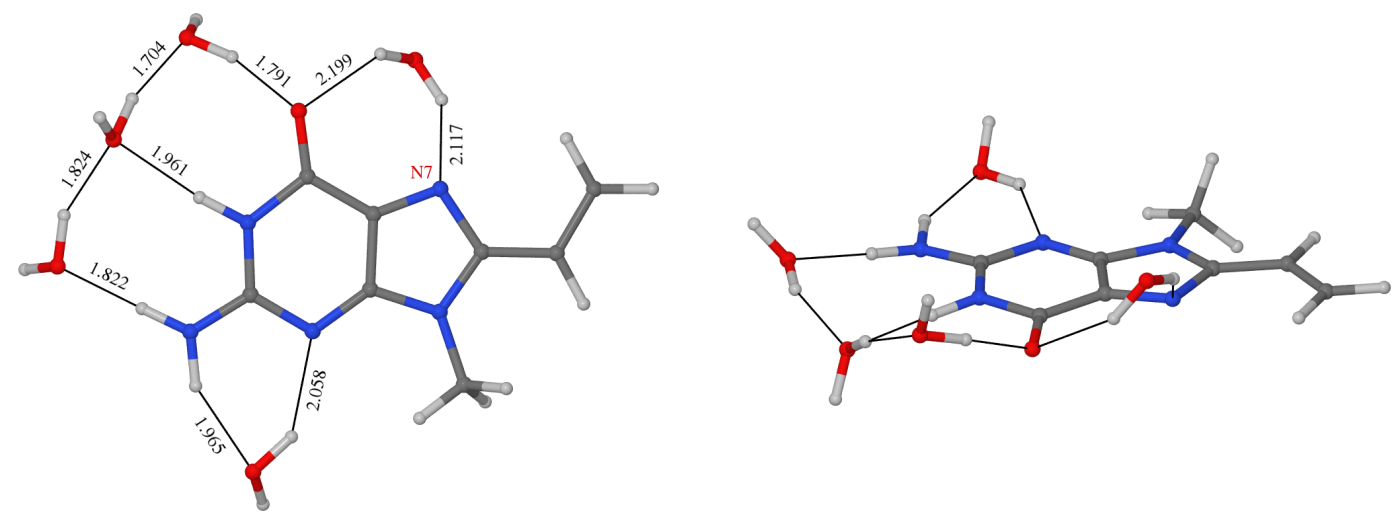

(b) EDDMs for the $\mathrm{S}_{1}$ and $\mathrm{S}_{2}$ adiabatic states of the $8 \mathrm{v} 9 \mathrm{MeG}-5 \mathrm{~W}$ complex at its $\mathrm{S}_{1}$-ICT-min geometry. The EDDMs are plotted in the form of isosurfaces with isovalues of $\pm 0.002 e / a_{0}{ }^{3}$. For each excited state, the red and blue isosurfaces delimit regions in which the electron density is increased and decreased, respectively, relative to the $\mathrm{S}_{0}$ state.

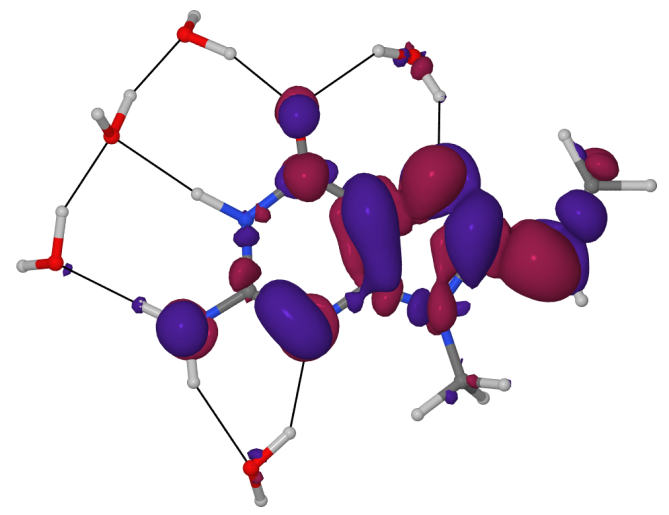

(i) $\mathrm{S}_{1}\left(\mathrm{ICT} \pi \pi^{*}\right)$

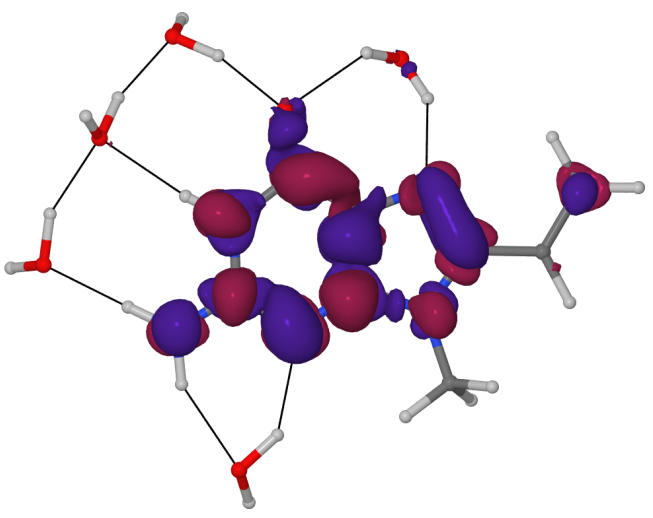

(ii) $\mathrm{S}_{2}\left(\mathrm{~L}_{\mathrm{a}} \pi \pi^{*}\right)$ 


\section{References}

[1] Stanton, J. F.; Bartlett, R. J. The Equation of Motion Coupled Cluster Method. A Systematic Biorthogonal Approach to Molecular Excitation Energies, Transition Probabilities, and Excited State Properties J. Chem. Phys. 1993, 98, 7029-7039.

[2] Szalay, P. G.; Watson, T.; Perera, A.; Lotrich, V. F.; Bartlett, R. J. Benchmark Studies on the Building Blocks of DNA. 1. Superiority of Coupled Cluster Methods in Describing the Excited States of Nucleobases in the Franck-Condon Region J. Phys. Chem. A 2012, 116, 6702-6710.

[3] Kánnár, D.; Szalay, P. G. Benchmarking coupled cluster methods on singlet excited states of nucleobases J. Mol. Model. 2014, 20, 2503.

[4] Hättig, C. Structure Optimizations for Excited States with Correlated Second-Order Methods: CC2 and ADC(2), in Advances in Quantum Chemistry, Vol. 50, Ed. H. J. Å. Jensen, Academic Press, New York, 2005, pp. 3760.

[5] MOLPRO is a package of ab initio programs written by H.-J. Werner, P. J. Knowles, G. Knizia, F. R. Manby, M. Schtz, P. Celani, T. Korona, R. Lindh, A. Mitrushenkov, G. Rauhut, K. R. Shamasundar, T. B. Adler, R. D. Amos, A. Bernhardsson, A. Berning, D. L. Cooper, M. J. O. Deegan, A. J. Dobbyn, F. Eckert, E. Goll, C. Hampel, A. Hesselmann, G. Hetzer, T. Hrenar, G. Jansen, C. Kppl, Y. Liu, A. W. Lloyd, R. A. Mata, A. J. May, S. J. McNicholas, W. Meyer, M. E. Mura, A. Nickla, D. P. O'Neill, P. Palmieri, D. Peng, K. Pflger, R. Pitzer, M. Reiher, T. Shiozaki, H. Stoll, A. J. Stone, R. Tarroni, T. Thorsteinsson, M. Wang. See http://www.molpro.net

[6] Mai, S.; Richter, M.; Marquetand, P.; González, L. Excitation of Nucleobases from a Computational Perspective II: Dynamics Top. Curr. Chem. 2015, 355, 99-154.

[7] Langer, H.; Doltsinis, N. L. Nonradiative Decay of Photoexcited Methylated Guanine Phys. Chem. Chem. Phys. 2004, 6, 2742-2748.

[8] Chen, H.; Li, S. Ab initio Study on Deactivation Pathways of Excited 9H-guanine J. Chem. Phys. 124, 2006, 154315.

[9] Serrano-Andrés, L.; Merchán, M.; Borin, A. C. A Three-State Model for the Photophysics of Guanine J. Am. Chem. Soc. 2008, 130, 2473-2484.

[10] Yamazaki, S.; Domcke, W.; Sobolewski, A. L. Nonradiative Decay Mechanisms of the Biologically Relevant Tautomer of Guanine J. Phys. Chem. A 2008, 112, 11965-11968.

[11] Yamazaki, S.; Domcke, W. Ab Initio Studies on the Photophysics of Guanine Tautomers: Out-of-Plane Deformation and $\mathrm{NH}$ Dissociation Pathways to Conical Intersections J. Phys. Chem. A 2008, 112, 7090-7097.

[12] Doltsinis, N. L., Markwick, P. R. L., Nieber, H., Langer, H. Ultrafast radiationless decay in nucleic acids: insights from nonadiabatic ab initio molecular dynamics. In: Radiation Induced Molecular Phenomena in Nucleic Acids. A Comprehensive Theoretical and Experimental Analysis; Shukla, M. K., Leszczynski, J., Eds.; Springer, 2008; pp 265-299.

[13] Lan, Z.; Fabiano, E.; Thiel, W. Photoinduced Nonadiabatic Dynamics of 9H-Guanine ChemPhysChem 2009, 10, 1225-1229.

[14] Barbatti, M.; Aquino, A. J. A.; Szymczak, J. J.; Nachtigallová, D.; Hobza, P.; Lischka, H. Relaxation Mechanisms of UV-photoexcited DNA and RNA Nucleobases Proc. Natl. Acad. Sci. USA 2010, 107, 21453-21458. 
[15] Barbatti, M.; Szymczak, J. J.; Aquino, A. J. A.; Nachtigallová, D.; Lischka, H. The Decay Mechanism of Photoexcited Guanine - A Nonadiabatic Dynamics Study J. Chem. Phys. 134, 2011, 014304

[16] Frank, I.; Hutter, J.; Marx, D.; Parrinello, M. Molecular Dynamics in Low-Spin Excited States J. Chem. Phys. 1998, 108, 4060.

[17] Evans, D. G.; Boeyens, J. C. A. Conformational Analysis of Ring Pucker Acta Cryst. 1989, B45, 581-590.

[18] Canuel, C.; Mons, M.; Piuzzi, F.; Tardivel, B.; Dimicoli, I.; Elhanine, M. Excited States Dynamics of DNA and RNA Bases: Characterization of a Stepwise Deactivation Pathway in the Gas Phase J. Chem. Phys. 2005, 122, 074316.

[19] Mons, M.; Dimicoli, I.; Piuzzi, F. Radiation induced molecular phenomena in nucleic acids: a comprehensive theoretical and experimental analysis, Isolated Guanine: Tautomerism, Spectroscopy and Excited State Dynamics, Springer, Dordrecht, London, 2008, pp. 343-367.

[20] Tuna, D.; Lefrancois, D.; Wolański, Ł.; Gozer, S.; Schapiro, I.; Andruniów, T.; Dreuw, A.; Olivucci, M. Assessment of Approximate Coupled-Cluster and Algebraic-Diagrammatic-Construction Methods for Ground- and Excited-State Reaction Paths and the Conical-Intersection Seam of a Retinal-Chromophore Model J. Chem. Theory Comput. 2015, 11, 5758-5781.

[21] Werner, H.-J. Third-Order Multireference Perturbation Theory. The CASPT3 Method Mol. Phys. 1996, 89, 645-661

[22] Ghigo, G.; Roos, B. O.; Malmqvist, P. A. A Modified Definition of the Zeroth-Order Hamiltonian in Multiconfigurational Perturbation Theory (CASPT2) Chem. Phys. Lett. 2004, $396,142-149$.

[23] Hill, A. D.; Reilly, P. J. Puckering Coordinates of Monocyclic Rings by Triangular Decomposition J. Chem. Inf. Model. 2007, 47, 1031-1035.

[24] Tully, J. C.; Preston, R. K. Trajectory Surface Hopping Approach to Nonadiabatic Molecular Collisions: The Reaction of $\mathrm{H}^{+}$with $\mathrm{D}_{2}$ J. Chem. Phys. 1971, 55, 562-572.

[25] Doltsinis, N. L.; Marx, D. First Principles Molecular Dynamics Involving Excited States and Nonadiabatic Transitions J. Theor. Comput. Chem. 2002, 1, 319-349.

[26] Tully, J. C. Molecular Dynamics with Electronic Transitions J. Chem. Phys. 1990, 93, 1061-1071.

[27] Plasser, F.; Crespo-Otero, R.; Pederzoli, M.; Pittner, J.; Lischka, H.; Barbatti, M. Surface Hopping Dynamics with Correlated Single-Reference Methods: 9H-Adenine as a Case Study J. Chem. Theory Comput. 2014, 10, 1395-1405.

[28] Barbatti, M.; Granucci, G.; Persico, M.; Ruckenbauer, M.; Vazdar, M.; Eckert-Maksic, M.; Lischka, H. The On-the-Fly Surface-Hopping Program System Newton-X: Application to $A b$ Initio Simulation of the Nonadiabatic Photodynamics of Benchmark Systems J. Photochem. Photobiol., A 2007, 190, 228-240.

[29] Barbatti, M.; Ruckenbauer, M.; Plasser, F.; Pittner, J.; Granucci, G.; Persico, M.; Lischka, H. Newton-X: a Surface-Hopping Program for Nonadiabatic Molecular Dynamics Wiley Interdiscip. Rev.: Comput. Mol. Sci. 2014, 4, 26-33.

[30] Butcher, J. A Modified Multistep Method for the Numerical Integration of Ordinary Differential Equations J. Assoc. Comput. Mach. 1965, 12, 124-135. 
[31] Crespo-Otero, R.; Barbatti, M. Spectrum Simulation and Decomposition with Nuclear Ensemble: Formal Derivation and Application to Benzene, Furan and 2-Phenylfuran Theor. Chem. Acc. 2012, 131, 1237.

[32] Gaussian 09, Revision A.02, Frisch, M. J.; Trucks, G. W.; Schlegel, H. B.; Scuseria, G. E.; Robb, M. A.; Cheeseman, J. R.; Scalmani, G.; Barone, V.; Mennucci, B.; Petersson, G. A.; Nakatsuji, H.; Caricato, M.; Li, X.; Hratchian, H. P.; Izmaylov, A. F.; Bloino, J.; Zheng, G.; Sonnenberg, J. L.; Hada, M.; Ehara, M.; Toyota, K.; Fukuda, R.; Hasegawa, J.; Ishida, M.; Nakajima, T.; Honda, Y.; Kitao, O.; Nakai, H.; Vreven, T.; Montgomery, J. A., Jr.; Peralta, J. E.; Ogliaro, F.; Bearpark, M.; Heyd, J. J.; Brothers, E.; Kudin, K. N.; Staroverov, V. N.; Kobayashi, R.; Normand, J.; Raghavachari, K.; Rendell, A.; Burant, J. C.; Iyengar, S. S.; Tomasi, J.; Cossi, M.; Rega, N.; Millam, J. M.; Klene, M.; Knox, J. E.; Cross, J. B.; Bakken, V.; Adamo, C.; Jaramillo, J.; Gomperts, R.; Stratmann, R. E.; Yazyev, O.; Austin, A. J.; Cammi, R.; Pomelli, C.; Ochterski, J. W.; Martin, R. L.; Morokuma, K.; Zakrzewski, V. G.; Voth, G. A.; Salvador, P.; Dannenberg, J. J.; Dapprich, S.; Daniels, A. D.; Farkas, Ö.; Foresman, J. B.; Ortiz, J. V.; Cioslowski, J.; Fox, D. J. Gaussian, Inc., Wallingford CT, 2009.

[33] Becke, A. D. Density-Functional Thermochemistry. III. The Role of Exact Exchange J. Chem. Phys. 1993, 98, 5648-5652.

[34] Stephens, P. J.; Devlin, F. J.; Chabalowski,C. F.; Frisch, M. J. Ab Initio Calculation of Vibrational Absorption and Circular Dichroism Spectra Using Density Functional Force Fields J. Phys. Chem. 1994, 98, 11623-11627.

[35] Weigend, F.; Ahlrichs, R. Balanced basis sets of split valence, triple zeta valence and quadruple zeta valence quality for $\mathrm{H}$ to Rn: Design and assessment of accuracy Phys. Chem. Chem. Phys. 2005, 7, 3297-3305.

[36] York, D. M.; Karplus, M. A Smooth Solvation Potential Based on the Conductor-Like Screening Model J. Phys. Chem. A 1999, 103, 11060.

[37] Scalmani, G.; Frisch, M. J. Continuous Surface Charge Polarizable Continuum Models of Solvation. I. General Formalism. J. Chem. Phys. 2010, 132, 114110.

[38] Papajak, E.; Zheng, J.; Xu, X.; Leverentz, H. R.; Truhlar, D. G. Perspectives on Basis Sets Beautiful: Seasonal Plantings of Diffuse Basis Functions J. Chem. Theory Comput. 2011, 7, 3027-3034. 